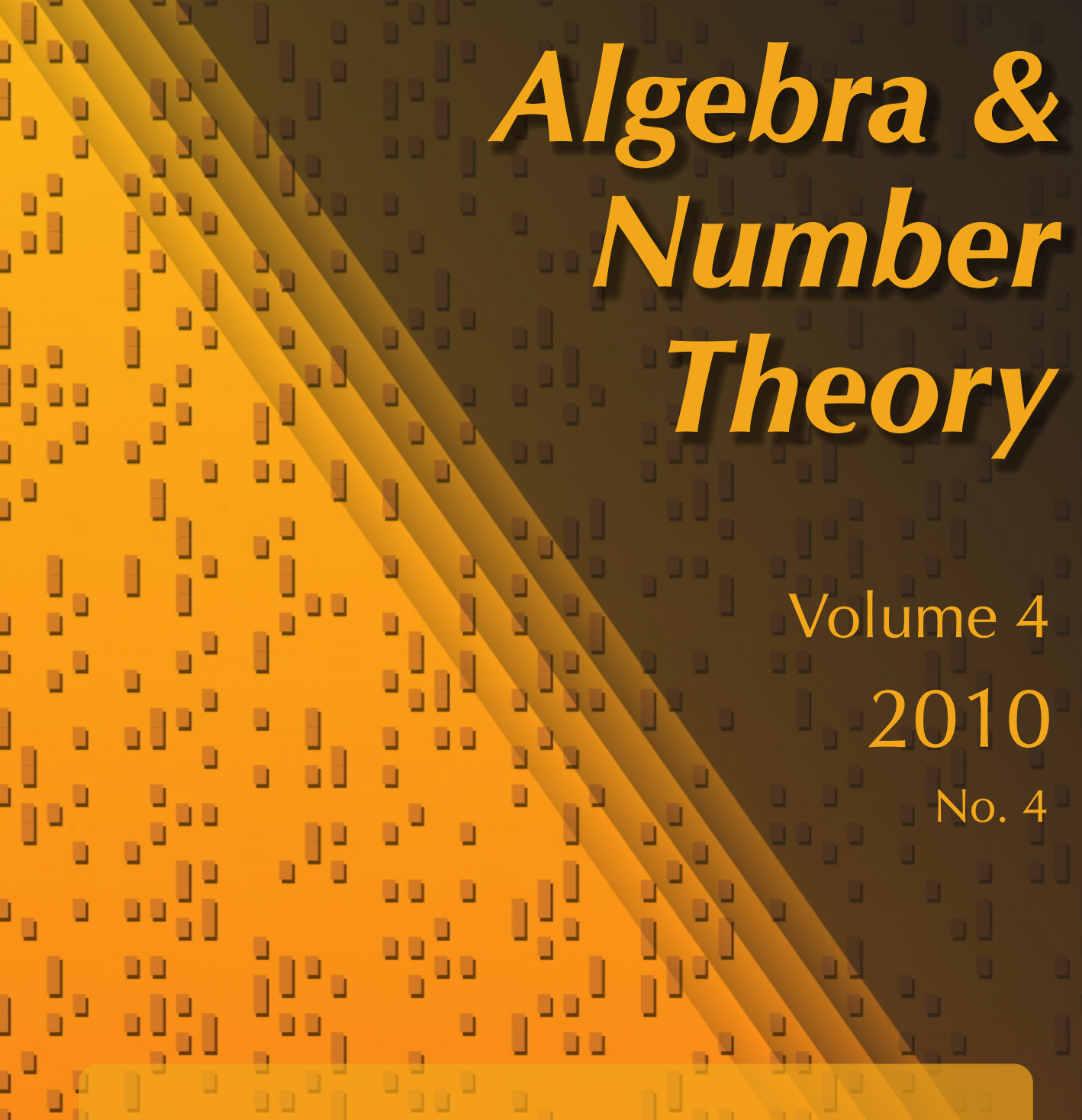

Algebraic properties of generic tropical varieties

Tim Römer and Kirsten Schmitz

\lrcorner

\rfloor

\lrcorner

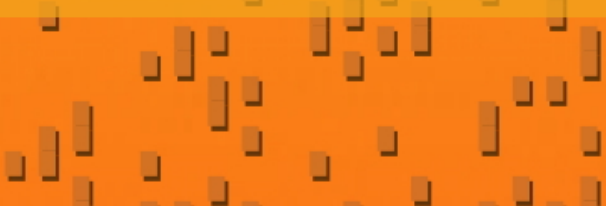

\lrcorner mathematical sciences pub fishers $\lrcorner\lrcorner\lrcorner\lrcorner$ 


\title{
Algebraic properties of generic tropical varieties
}

\author{
Tim Römer and Kirsten Schmitz
}

\begin{abstract}
We show that the algebraic invariants multiplicity and depth of the quotient ring $S / I$ of a polynomial ring $S$ and a graded ideal $I \subset S$ are closely connected to the fan structure of the generic tropical variety of $I$ in the constant coefficient case. Generically the multiplicity of $S / I$ is shown to correspond directly to a natural definition of multiplicity of cones of tropical varieties. Moreover, we can recover information on the depth of $S / I$ from the fan structure of the generic tropical variety of $I$ if the depth is known to be greater than 0 . In particular, in this case we can see if $S / I$ is Cohen-Macaulay or almost-Cohen-Macaulay from the generic tropical variety of $I$.
\end{abstract}

\section{Introduction}

As a very new area of mathematics, tropical geometry has received a lot of attention, from various points of view, in the last few years; see [Develin and Sturmfels 2004; Katz et al. 2008; Mikhalkin 2006; Speyer and Sturmfels 2004; Gathmann 2006; Itenberg et al. 2007] for review articles. One approach to tropical geometry, which provides an effective tool for studying questions in algebraic geometry, is to associate a combinatorial object to a projective algebraic variety; see for example [Draisma 2008; Gathmann and Markwig 2008]. More precisely, the tropical variety $T(X)$ of an algebraic variety $X$ is the real-valued image of $X$ under some valuation map [Draisma 2008; Jensen et al. 2008; Speyer and Sturmfels 2004]. In certain settings, $T(X)$ has the structure of a polyhedral complex [Bieri and Groves 1984; Jensen 2007], and there is a practical characterization in terms of initial ideals given in [Speyer and Sturmfels 2004; Draisma 2008, Theorem 4.2]. If the valuation on the ground field is trivial, $T(X)$ is a subfan of the Gröbner fan of the ideal $I$ defining $X$. We only consider this constant coefficient case, and we define the tropical variety as a fan associated to $I$ instead of $X$. In this situation the ideal $I$ need not be a radical ideal. So let $K$ be an infinite field and $K\left[x_{1}, \ldots, x_{n}\right]$ be the

MSC2000: primary 13F20; secondary 14Q99, 13P10.

Keywords: tropical variety, constant coefficient case, Gröbner fan, generic initial ideals,

Cohen-Macaulay, multiplicity, depth. 
polynomial ring in $n$ variables over $K$. In this setting, the tropical variety $T(I)$ of a graded ideal $I \subset K\left[x_{1}, \ldots, x_{n}\right]$ is defined to be the subfan of the Gröbner fan of $I$ that consists of all cones such that the corresponding initial ideal does not contain a monomial.

The tropical variety of an ideal depends on the choice of coordinates in the following sense. For $g \in \mathrm{GL}_{n}(K)$, the image $g(I)$ of a graded ideal $I \subset S=$ $K\left[x_{1}, \ldots, x_{n}\right]$ is also a graded ideal, and all important algebraic invariants of $S / I$, such as the dimension, multiplicity and depth, are preserved under $g$. In fact, $g(I)$ can be considered as the ideal $I$ given in different coordinates. In general, for $I \subset K\left[x_{1}, \ldots, x_{n}\right]$ and $g \in \mathrm{GL}_{n}(K)$, we have

$$
T(I) \neq T(g(I)) .
$$

We can, however, find a nonempty Zariski-open set $U \subset \mathrm{GL}_{n}(K)$ such that $T(g(I))$ is the same fan $\mathrm{gT}(I)$ for every $g \in U$ [Römer and Schmitz 2009, Corollary 6.7]. The fact that $U$ is dense in $\mathrm{GL}_{n}(K)$ justifies the name generic tropical variety of $I$ for this fan. In Corollary 7.4 of the same reference it was shown that $\mathrm{gT}(I)$ as a set depends only on the dimension of $S / I$. More precisely, if $S / I$ is $m$-dimensional, the underlying set of $\mathrm{gT}(I)$ is always the $m$-skeleton of a particular complete fan $\mathcal{W}_{n}$ in $\mathbb{R}^{n}$. We show that under certain conditions, we can recover information on the depth of $S / I$ in addition to the dimension from the fan structure of $\mathrm{gT}(I)$ induced by the Gröbner fan. As one of the main results, we can completely describe generic tropical varieties as fans if $S / I$ is Cohen-Macaulay or almost-Cohen-Macaulay. With this we can determine if $S / I$ is Cohen-Macaulay or almost-Cohen-Macaulay from the fan structure of the generic tropical variety of $I$ if we know the depth of $S / I$ to be greater than 0 . Moreover, we show that the multiplicities associated with the maximal cones of $\mathrm{gT}(I)$ as done in [Dickenstein et al. 2007] correspond directly to the multiplicity of $S / I$.

Our paper is organized as follows. In Section 2 we introduce basic results and necessary notation. In Section 3 we show that for an $m$-dimensional ring $S / I$ for a graded ideal $I$, the generic tropical variety is always a subfan of the $m$-skeleton of $W_{n}$ by showing that the fan structure induced by $\mathscr{W}_{n}$ is the coarsest possible on the underlying set. This will be important in all following sections. Sections 4 and 5 are devoted to the depth of $S / I$. In Section 4 we show that $g T(I)$ is equal to the $m$ skeleton of $W_{n}$ if and only if $S / I$ is Cohen-Macaulay or almost-Cohen-Macaulay, where $\operatorname{dim}(S / I)=m$. In Section 5 we show that we can recover the depth of $S / I$ from $\mathrm{gT}(I)$ if we know it to be greater than 0 and less than $\operatorname{dim}(S / I)-1$. We also give more structural results depending on $\operatorname{depth}(S / I)$ on $\operatorname{gT}(I)$ as a fan for a special class of ideals. We show in Section 6 that the multiplicities defined on the maximal cones of $T(I)$ as in [Dickenstein et al. 2007] generically behave in a nice way. These multiplicities coincide with the multiplicity of $S / I$. 


\section{Preliminaries}

Let $K$ be an algebraically closed field of characteristic 0 and let $S=K\left[x_{1}, \ldots, x_{n}\right]$ be the polynomial ring in $n$ variables over $K$. The $\omega$-weight $\mathrm{wt}_{\omega}\left(c x^{\nu}\right)$ of some term $c x^{\nu}=c x_{1}^{\nu_{1}} \cdots x_{n}^{\nu_{n}} \in S$ is defined as $\mathrm{wt}_{\omega}\left(c x^{\nu}\right)=\omega \cdot v$ for any $\omega \in \mathbb{R}^{n}$. For a homogeneous polynomial $f \in S$ with $f=\sum_{\nu \in \mathbb{N}^{n}} a_{v} x^{v}$ and $\omega \in \mathbb{R}^{n}$, the initial polynomial $\operatorname{in}_{\omega}(f)$ of $f$ consists of all terms of $f$ whose $\omega$-weight $\omega \cdot v$ is minimal. We use multiplicative term orders $\succ$ on the monomials of $S$ and define $\operatorname{in}_{\succ}(f)$ to be the term $c x^{\nu}$ of $f$ for which $c x^{\nu} \succ d x^{\mu}$ for every other term $d x^{\mu}$ of $f$. For $\omega \in \mathbb{R}^{n}$ and a term order $\succ$ we can consider the refinement $\succ_{\omega}$. This is the term order that first compares terms by their $\omega$-weight and uses $\succ$ to break ties. Note that while initial polynomials with respect to $\omega$ are defined by taking terms of minimal $\omega$-weight, the symbol $\succ$ suggests that $\operatorname{in}_{\succ}(f)$ is the "largest" term of $f$. The reason for considering this counterintuitive setup is that in Gröbner basis theory one usually considers the largest terms as initial terms, while in tropical geometry it is convenient to work with the minimal $\omega$-weight.

In particular, we will repeatedly need a (degree) reverse lexicographic term order. With respect to an ordering $x_{j_{1}} \succ x_{j_{2}} \succ \cdots \succ x_{j_{n}}$ of the variables, this monomial order is defined as follows. For $\nu, \mu \in\left(\mathbb{N}_{0}\right)^{n}$ we have $x^{\nu} \succ x^{\mu}$ if either $\sum_{i} v_{i}>\sum_{i} \mu_{i}$ or $\sum_{i} v_{i}=\sum_{i} \mu_{i}$ and there exists $k \in\{1, \ldots, n\}$ such that $v_{j_{i}}=\mu_{j_{i}}$ for $i>k$ and $v_{j_{k}}<\mu_{j_{k}}$. Since we only consider graded ideals, we will simply call this order a reverse lexicographic order. If no ordering of the variables is specified, we mean the reverse lexicographic order with respect to $x_{1} \succ x_{2} \succ \cdots \succ x_{n}$.

We consider graded ideals $I \subset S$ and always assume $I \neq(0)$ if not stated otherwise. The dimension $\operatorname{dim}(S / I)$ refers to the Krull dimension of the ring $S / I$. Since we assume $I \neq(0)$, we always have $\operatorname{dim}(S / I)<n$. The initial ideal of $I \subset S$ with respect to $\omega \in \mathbb{R}^{n}$ is defined as

$$
\operatorname{in}_{\omega}(I)=\left(\operatorname{in}_{\omega}(f): f \in I\right) .
$$

For $I \subset S=K\left[x_{1}, \ldots, x_{n}\right]$, we define the tropical variety of $I$ by

$$
T(I)=\left\{\omega \in \mathbb{R}^{n}: \operatorname{in}_{\omega}(I) \text { does not contain a monomial }\right\} .
$$

This is a special case, called the constant coefficient case, of the usual definition of a tropical variety as the image of a projective variety under a valuation map; see for example [Draisma 2008; Speyer and Sturmfels 2004]. In this case, $K$ is considered to have a trivial valuation [Draisma 2008, Theorem 4.2]. Then the tropical variety $T(I)$ is a subfan of the Gröbner fan $\mathrm{GF}(I)$ of $I$ as observed in [Bogart et al. 2007]. Recall that the Gröbner fan is a complete fan in $\mathbb{R}^{n}$, where $\omega, \omega^{\prime} \in \mathbb{R}^{n}$ are in the same relatively open cone if $\operatorname{in}_{\omega}(I)=\operatorname{in}_{\omega^{\prime}}(I)$; see for example [Mora and Robbiano 1988; Sturmfels 1996]. Sometimes we denote the ideal $\operatorname{in}_{\omega}(I)$ for a relatively open cone $\stackrel{\circ}{C}$ of $\operatorname{GF}(I)$ and $\omega \in \stackrel{\circ}{C}$ by $\operatorname{in}_{C}(I)$. 
We study the structure of the tropical variety under a generic coordinate transformation in the following sense. For $g \in \mathrm{GL}_{n}(K)$, we regard the $K$-algebra automorphism induced by

$$
\begin{aligned}
K\left[x_{1}, \ldots, x_{n}\right] & \rightarrow K\left[x_{1}, \ldots, x_{n}\right], \\
x_{i} & \mapsto \sum_{j=1}^{n} g_{j i} x_{j} .
\end{aligned}
$$

In the sequel, we identify $g$ with this automorphism and call both of them $g$. Note that this definition differs from [Römer and Schmitz 2009, Definition 2.4] by a transposition of the matrix $g$. However, this does not affect the results proved in that paper. We consider $\mathrm{GL}_{n}(K)$ equipped with the Zariski-topology. If $S / I$ is 0-dimensional, then for every $g \in \mathrm{GL}_{n}(K)$, the tropical variety $T(g(I))$ is empty [Römer and Schmitz 2009, Lemma 2.5]. We will therefore always assume that $\operatorname{dim}(S / I)>0$. In Corollary 6.9 of the same work it was shown that for a graded ideal $I \subset S=K\left[x_{1}, \ldots, x_{n}\right]$ with $\operatorname{dim}(S / I)>0$, there exists a Zariski-open set $\varnothing \neq U \subset \mathrm{GL}_{n}(K)$ such that $T(g(I))$ is the same fan for every $g \in U$. This fan is denoted by $\mathrm{gT}(I)$ and called the generic tropical variety of $I$. If $g \in U$, then $g(I)$ is called a generic coordinate transformation of $I$. Moreover, by [Römer and Schmitz 2009, Theorem 3.1] we know that there is also a generic Gröbner fan $\operatorname{gGF}(I)$ such that $\operatorname{GF}(g(I))=\operatorname{gGF}(I)$ as a fan for every $g \in U$. The monomial initial ideal $\operatorname{in}_{\succ}(g(I))$ with respect to a term order $\succ$ is exactly the generic initial ideal $\operatorname{gin}_{\succ}(I)$ for $g \in U$. These generic initial ideals correspond to the maximal cones of $\operatorname{gGF}(I)$. In the following, we will fix a nonempty Zariski-open subset $U \subset \mathrm{GL}_{n}(K)$ such that

$$
\mathrm{GF}(g(I))=\operatorname{gGF}(I) \quad \text { and } \quad T(g(I))=\operatorname{gT}(I) \quad \text { for every } g \in U,
$$

and refer to it simply as $U$.

The generic tropical variety as a set is always equal to some skeleton of a particular complete fan $\mathcal{W}_{n}$ in $\mathbb{R}^{n}$. We recall that this fan is defined by the maximal cones $C_{i}=\left\{\omega \in \mathbb{R}^{n}: \omega_{i}=\min _{k}\left\{\omega_{k}\right\}\right\}$ for $i=1, \ldots, n$. Note that to define a fan in $\mathbb{R}^{n}$ or to show that two fans in $\mathbb{R}^{n}$ are the same, it suffices to do this for the maximal cones. This is because every cone in a fan is a face of a maximal cone, so all cones in a fan are determined by the maximal cones. Every $m$-dimensional cone $C_{A}$ in $W_{n}$ for $m \in\{1, \ldots, n\}$ has the form $C_{A}=\left\{\omega \in \mathbb{R}^{n}: \omega_{i}=\min _{k}\left\{\omega_{k}\right\}\right.$ for $\left.i \in A\right\}$, where $A \subset\{1, \ldots, n\}$ with $|A|=n-m+1$. On the other hand, every nonempty $A \subset\{1, \ldots, n\}$ defines a cone of $\mathscr{W}_{n}$ in this way, which we will denote by $C_{A}$. We let $\mathcal{W}_{n}^{m}$ be the $m$-skeleton of $\mathcal{W}_{n}$, that is, the fan consisting of all cones of $\mathcal{W}_{n}$ of dimension less than or equal to $m$. In [Römer and Schmitz 2009, Corollary 7.4], it was shown that for a graded ideal $I \subset S=K\left[x_{1}, \ldots, x_{n}\right]$ with $\operatorname{dim}(S / I)=m$, the generic tropical variety $\mathrm{gT}(I)$ coincides with $W_{n}^{m}$ as a set. 
For a fan $\mathscr{F}$ in $\mathbb{R}^{n}$, we denote by $|\mathscr{F}|$ the set $\bigcup_{C \in \mathscr{F}} C$ (without its fan structure), where the union is taken over all cones of $\mathscr{F}$. The notation $C$ for a cone of $\mathscr{F}$ always refers to a closed cone. By $\stackrel{\circ}{C}$ we denote the relative interior of $C$. We say that a fan $\mathscr{E}$ in $\mathbb{R}^{n}$ refines a fan $\mathscr{F}$ in $\mathbb{R}^{n}$ if $|\mathscr{E}|=|\mathscr{F}|$ and for every relatively open cone $\stackrel{\circ}{C}$ of $\mathscr{E}$ there exists a relatively open cone $\stackrel{\circ}{D}$ of $\mathscr{F}$ with $\stackrel{\circ}{C} \subset \stackrel{\circ}{D}$. In Proposition 3.5 we show that it suffices to check this condition for the maximal cones of $\mathscr{E}$.

\section{Fan structures on the set $\left|W_{n}^{m}\right|$}

In this section we assume $0<m<n$. The aim is to show that $\mathcal{W}_{n}^{m}$ is the coarsest fan structure on the set $\left|W_{n}^{m}\right|$. By this we mean that every fan $\mathscr{F}$ in $\mathbb{R}^{n}$ with $\left|\mathscr{F}^{\prime}\right|=\left|W_{n}^{m}\right|$ refines $\mathcal{W}_{n}^{m}$ as a fan. We first prove that any fan on $\left|\mathcal{W}_{n}^{m}\right|$ is pure, by proving this statement for any subset of $\mathbb{R}^{n}$ that permits a pure fan structure of dimension at most $n-1$. We repeatedly need the following lemma.

Lemma 3.1. Let $\mathscr{F}$ be a fan in $\mathbb{R}^{n}$ and $C$ a cone of $\mathscr{F}$. Let $\omega \in \stackrel{\circ}{\mathrm{C}}$ and $\left(\omega_{i}\right)_{i \in \mathbb{N}}$ be a sequence such that $\omega_{i} \in|\mathscr{F}| \backslash C$ and $\lim _{i \rightarrow \infty} \omega_{i}=\omega$. Then there exists a cone $D$ in $\mathscr{F}$ containing a subsequence of $\left(\omega_{i}\right)_{i \in \mathbb{N}}$ such that $C$ is a proper face of $D$.

Proof. Since $\omega_{i} \in \mathscr{F}$, there exists some other cone $C_{i} \neq C$ such that $\omega_{i} \in \stackrel{C}{C}_{i}$. But $\mathscr{F}$ has only finitely many cones, so there exists a subsequence $\left(\omega_{j_{i}}\right)_{j_{i} \in \mathbb{N}}$ of $\left(\omega_{i}\right)_{i \in \mathbb{N}}$ such that $\omega_{j_{i}} \in D$ for one particular cone $D$ of $\mathscr{F}$. By the choice of $\omega_{i}$, we have $D \neq C$. Now $\lim _{i \rightarrow \infty} \omega_{j_{i}}=\omega$ and $D$ is closed, so $\omega \in D$. Because $\stackrel{\circ}{\cap} \cap \stackrel{\circ}{D}=\varnothing$, we have $\omega \in \partial D$. By assumption, $C$ and $D$ intersect in a face of both of them. Since $\omega \in \stackrel{\circ}{\mathrm{C}}$ is in this intersection, this face is $C$. Hence, $C \subsetneq D$ as a face.

With this we can show in the following proposition that any fan structure on $\left|W_{n}^{m}\right|$ is pure.

Proposition 3.2. Let $\mathscr{E}$ be a pure $m$-dimensional fan in $\mathbb{R}^{n}$ and $\mathscr{F}$ an arbitrary fan in $\mathbb{R}^{n}$ with $|\mathscr{F}|=|\mathscr{E}|$. Then $\mathscr{F}$ is also a pure $m$-dimensional fan.

Proof. Let $C$ be any cone in $\mathscr{F}$. Assume that $\operatorname{dim} C<m$ and let $\omega \in \stackrel{\circ}{C}$. Since for any open neighborhood $W(\omega) \subset \mathbb{R}^{n}$ of $\omega$ we have $\operatorname{dim} W(\omega) \cap C<m$, there always exists $v \in(W(\omega) \cap|\mathscr{F}|) \backslash C$. So if we choose a sequence $\left(\varepsilon_{n}\right)_{n \in \mathbb{N}}$ with $\varepsilon_{n}>0$ for every $n \in \mathbb{N}$ and $\lim _{n \rightarrow \infty} \varepsilon_{n}=0$, there exists $v_{n} \in|\mathscr{F}| \backslash C$ with $\left|v_{n}-\omega\right|<\varepsilon_{n}$. By Lemma 3.1, we obtain a cone $D$ of $\mathscr{F}$ such that $C \subsetneq D$. Since $\operatorname{dim} D>\operatorname{dim} C$, either the proof is complete if $\operatorname{dim} D=m$, or we can apply the same procedure to $D$ instead of $C$. Either way, we obtain an $m$-dimensional cone of which $C$ is a face after finitely many steps.

This immediately implies the following corollary, which is a generalization of the fact that the tropical variety of a prime ideal $P$ with $\operatorname{dim}(S / P)=m$ that does not contain a monomial is a pure $m$-dimensional fan [Bieri and Groves 1984]. 
Corollary 3.3. Let $I \subset S=K\left[x_{1}, \ldots, x_{n}\right]$ be a graded ideal with $\operatorname{dim}(S / I)=m$. Then $\mathrm{gT}(I)$ is a pure $m$-dimensional fan.

To prove that a fan $\mathscr{E} \subset \mathbb{R}^{n}$ refines another fan $\mathscr{F} \subset \mathbb{R}^{n}$, it suffices to consider the maximal cones of $\mathscr{E}$. This will be the result of the next two statements.

Lemma 3.4. Let $D, C$ be cones in $\mathbb{R}^{n}$ such that $D \subset C$ and $D \cap \stackrel{\circ}{0} \neq \varnothing$. Then $\stackrel{\perp}{D} \subset \stackrel{\circ}{C}$.

Proof. Let $p \in \stackrel{\circ}{D}$, and for some $\varepsilon>0$ let $W=\{u \in \stackrel{\circ}{D}:|u-p|<\varepsilon\} \subset \stackrel{D}{D}$ be a relatively open neighborhood of $p$ in $\stackrel{\circ}{D}$. If $p \in \partial C$, then there exists a face $F$ of $C$ with $p \in F$. Let $H=\left\{\omega \in \mathbb{R}^{n}: a \cdot \omega=0\right\}$ be a defining hyperplane of $F$, so $F=H \cap C$, and let $C \subset H^{-}=\left\{\omega \in \mathbb{R}^{n}: a \cdot \omega \leq 0\right\}$. Since $W \subset \stackrel{D}{ } \subset C$, we know that $a \cdot u \leq 0$ for every $u \in W$. In addition, we have $a \cdot p=0$, because $p \in F$. Assume there exists $u \in W$ such that $a \cdot u<0$. Then we can choose $0<\lambda<1$ very small such that $p+\lambda(p-u) \in \stackrel{\circ}{D}$. Moreover, $|(p+\lambda(p-u))-p|=|\lambda(p-u)|<\varepsilon$, so $p+\lambda(p-u) \in W$. But $(p+\lambda(p-u)) \cdot a=-\lambda u \cdot a>0$, which is a contradiction to $p+\lambda(p-u) \in C$. Hence, $a \cdot u=0$ for every $u \in W$. Thus $W \subset H$, and since $W$ is relatively open in $D$, we also have $\operatorname{aff}(D) \subset H$. But then

$$
D \subset \operatorname{aff}(D) \cap C \subset H \cap C=F,
$$

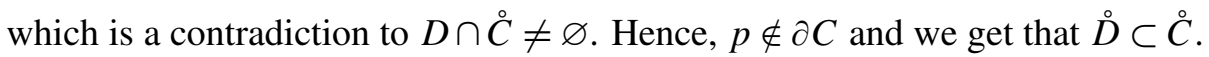

Proposition 3.5. Let $\mathscr{E}, \mathscr{F} \subset \mathbb{R}^{n}$ be two fans. Then $\mathscr{E}$ refines $\mathscr{F}$ as a fan if and only if $|\mathscr{E}|=|\mathscr{F}|$ and for every maximal cone $C \subset \mathscr{E}$ there exists a cone $D \subset \mathscr{F}$ such that $\stackrel{\circ}{C} \subset \stackrel{\circ}{D}$.

Proof. One implication follows directly from the definition of refinement. For the other one, we have to show that for any cone $K \subset \mathscr{E}$, there exists a cone $L \subset \mathscr{F}$ such that $\stackrel{\circ}{K} \subset \stackrel{\circ}{L}$. If $K$ is maximal, this is true by assumption. Let $K \subset \mathscr{E}$ be not maximal. Then there exists a maximal cone $C \subset \mathscr{E}$ such that $K$ is a face of $C$. Moreover, we know that $\stackrel{\circ}{C} \subset \stackrel{\circ}{D}$ for some cone $D \in \mathscr{F}$. So $K \subset D$. Assume that

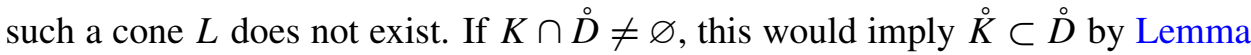
3.4 and we could set $L=D$. Hence, $K \cap \stackrel{D}{D}=\varnothing$. Then $K \subset \partial D$ and by [Bruns and Gubeladze 2009, Lemma 1.5], it follows that $K \subset E$ for a proper face $E$ of $D$. Since $\operatorname{dim} E<\operatorname{dim} D$, we can use a suitable induction to obtain a sequence of cones in $\mathscr{F}$ of strictly decreasing dimension such that $K$ does not intersect the relative interior of each cone. The last cone in this sequence has to be the lineality space $A$ of $\mathscr{F}$. So by this induction, we get $K \subset \partial A$, which is a contradiction to $\partial A=\varnothing$. Hence, there has to exist a cone $L \subset \mathscr{F}$ such that $\stackrel{\circ}{K} \subset \stackrel{\circ}{L}$.

The proof of the next auxiliary result is elementary, so we omit it. 
Lemma 3.6. Let $C \subset \mathbb{R}^{n}$ be a cone and let $\operatorname{dim} C=m$. Also, let $D_{1}, \ldots, D_{s} \subset \mathbb{R}^{n}$ be cones such that

$$
C \subset \bigcup_{i=1}^{s} D_{i},
$$

where $\operatorname{dim} D_{1}=m$ and $\operatorname{dim} D_{2}, \ldots, \operatorname{dim} D_{s}<m$. Then $C \subset D_{1}$.

With these prerequisites, we can show that for $m<n$, the fan structure of $\mathscr{W}_{n}^{m}$ is actually the coarsest possible on the set $\left|W_{n}^{m}\right|$ in the sense that every other fan $\mathscr{F} \subset$ $\mathbb{R}^{n}$ with $\left|\mathscr{F}_{\mid}\right|=\left|W_{n}^{m}\right|$ refines $\mathscr{W}_{n}^{m}$ as a fan. In particular, this will imply that $\mathrm{gT}(I)$ refines $\mathcal{W}_{n}^{m}$ as a fan for a graded ideal $I \subset S=K\left[x_{1}, \ldots, x_{n}\right]$ with $\operatorname{dim}(S / I)=m$.

Proposition 3.7. Let $m<n$ and $\mathscr{F} \subset \mathbb{R}^{n}$ be a fan with $|\mathscr{F}|=\left|W_{n}^{m}\right|$. Then for every relatively open cone $\stackrel{\circ}{C}$ of $\mathscr{F}$, there exists a relatively open cone $\stackrel{\circ}{C}_{A}$ of $\mathscr{W}_{n}^{m}$ such that $\stackrel{\circ}{\mathrm{C}} \subset \stackrel{\circ}{C}_{A}$.

Proof. ${ }^{W_{n}^{m}}=\dot{U}_{|A| \geq n-m+1} \stackrel{\circ}{C}_{A}$ is the disjoint union of all relatively open cones of $\mathscr{W}_{n}$ whose defining set $A \subset\{1, \ldots, n\}$ has at least $n-m+1$ elements. By Proposition 3.5 it suffices to prove the condition for the maximal cones of $\mathscr{F}$. Let $C$ be a maximal cone of $\mathscr{F}$. Then $\operatorname{dim} C=m$, as $\mathscr{F}$ is pure by Proposition 3.2. Since $\operatorname{dim} \bigcup_{|A|>n-m+1} \stackrel{\circ}{C}_{A}=m-1<m$, there exists an $\omega \in \dot{C}$ that is contained in the interior of some maximal cone $\stackrel{\circ}{C}_{A_{1}}$ of ${ }^{\mathcal{W}}{ }_{n}^{m}$ with $\left|A_{1}\right|=n-m+1$. Assume there exists $v \in \stackrel{\circ}{C}$ such that $v \in \stackrel{\circ}{C}_{A_{2}}$ for a different maximal cone $\stackrel{\circ}{C}_{A_{2}}$ of $\mathscr{W}_{n}^{m}$. Then $\left|A_{1} \cap A_{2}\right|<n-m+1$. We have to consider two cases:

- If $A_{1} \cap A_{2} \neq \varnothing$, the minimal coordinates of $\omega+v$ are attained exactly at the indices in $A_{1} \cap A_{2}$. But $\left|A_{1} \cap A_{2}\right|<n-m+1$, so $\omega+v \notin\left|W_{n}^{m}\right|$. This is a contradiction to $\omega+v \in \stackrel{\circ}{C} \subset\left|W_{n}^{m}\right|$.

- Assume that $A_{1} \cap A_{2}=\varnothing$. Since $\operatorname{dim} C=m$, we can change the coordinates of $\omega$ that are not contained in $A_{1}$ independently from each other by adding or subtracting small real numbers without leaving $\stackrel{\odot}{C}$. The same is true for the coordinates of $v$ that are not in $A_{2}$. Hence, we can change every coordinate of $\omega+v$ by a small amount without leaving $\stackrel{\circ}{C}$, since $A_{1} \cap A_{2}=\varnothing$. But then we can assume that the minimum of the coordinates of $\omega+v$ is attained only once. Again we have $\omega+v \notin\left|W_{n}^{m}\right|$, contradicting $\omega+v \in \stackrel{\circ}{C} \subset\left|W_{n}^{m}\right|$.

Hence, no element of $\stackrel{\circ}{C}$ can be contained in the relative interior of any maximal cone of $\mathcal{W}_{n}^{m}$ other than $C_{A_{1}}$. But then

$$
\stackrel{\circ}{C} \subset \stackrel{\circ}{C}_{A_{1}} \cup\left(\bigcup_{|A|>n-m+1} \stackrel{\circ}{C}_{A}\right) .
$$

Taking the topological closure, this implies $C \subset C_{A_{1}}$ by Lemma 3.6. Since both cones have the same dimension, we also have $\stackrel{\circ}{C} \subset \stackrel{\circ}{C}_{A_{1}}$ by Lemma 3.4. 
Proposition 3.7 implies as a corollary that the generic tropical variety always refines $\mathcal{W}_{n}^{m}$ as a fan.

Corollary 3.8. Let $I \subset S=K\left[x_{1}, \ldots, x_{n}\right]$ be a graded ideal with $\operatorname{dim}(S / I)=m>$ 0 . Then for every relatively open cone $\stackrel{\circ}{C}$ of $\mathrm{gT}(I)$, there exists a relatively open cone $\stackrel{\circ}{D}$ of ${ }^{\text {W }}{ }_{n}^{m}$ such that $\stackrel{\circ}{\mathrm{C}} \subset \stackrel{\circ}{\mathrm{D}}$.

Proof. Since $|\mathrm{gT}(I)|=\left|W_{n}^{m}\right|$ by [Römer and Schmitz 2009, Corollary 7.4] and $W_{n}^{m}$ is pure $m$-dimensional, it follows from Proposition 3.2 that $\mathrm{gT}(I)$ is a pure $m$-dimensional fan. The claim now is a consequence of Proposition 3.7.

\section{Generic tropical varieties of Cohen-Macaulay and almost-Cohen-Macaulay rings}

In addition to the dimension of $S / I$, it is also possible to recover information on the depth of $S / I$ from the generic tropical variety of $I$. We will show that for an ideal $I$ with $\operatorname{dim}(S / I)=m$ and $\operatorname{depth}(S / I)>0$, the generic tropical variety is $\mathcal{W}_{n}^{m}$ as a fan if and only if $\operatorname{depth}(S / I)=\operatorname{dim}(S / I)$ or $\operatorname{depth}(S / I)=\operatorname{dim}(S / I)-1$. Thus we can read off whether $S / I$ is Cohen-Macaulay or almost-Cohen-Macaulay from the fan $\mathrm{gT}(I)$.

To define the depth of $S / I$, recall that a system of linear forms $l_{1}, \ldots, l_{t} \in S / I$ is called a regular sequence for $S / I$ if $l_{i}$ is not a zero-divisor on $(S / I) /\left(l_{1}, \ldots, l_{i-1}\right)$ for $i=1, \ldots, t$.

Definition 4.1. For a graded ideal $I \subset S=K\left[x_{1}, \ldots, x_{n}\right]$, we define the depth of $S / I$ to be

$$
\operatorname{depth}(S / I)=\max \left\{\begin{array}{c}
t \in \mathbb{N}: \text { there is a regular sequence } \\
\text { of linear forms } l_{1}, \ldots, l_{t} \in S / I
\end{array}\right\} .
$$

The depth is bounded from above by the dimension of $S / I$; see for example [Bruns and Herzog 1993, Proposition 1.2.12]. Also, we know that depth $(S / I) \geq$ $\operatorname{depth}\left(S / \operatorname{gin}_{\succ}(I)\right)$ for any term order $\succ$. Equality holds if $\succ$ is a reverse lexicographic order with respect to some ordering of the variables. These two statements follow from [Bruns and Conca 2004, Corollary 3.5 and Remark 3.6] together with the Auslander-Buchsbaum formula.

In general it is not possible to see the depth of $S / I$ in the fan $T(I)$, as the following example shows.

Example 4.2. For $1 \leq k \leq n$, consider the ideal

$$
I=\left(x_{1}\left(x_{1}+x_{2}\right), x_{2}\left(x_{1}+x_{2}\right), \ldots, x_{k}\left(x_{1}+x_{2}\right)\right) \subset S=K\left[x_{1}, \ldots, x_{n}\right] .
$$

Then $\operatorname{dim}(S / I)=n-1$ and $\operatorname{depth}(S / I)=n-k$. But the tropical variety $T(I)$ always consists of only one cone $T(I)=\left\{\omega \in \mathbb{R}^{n}: \omega_{1}=\omega_{2}\right\}$ which is independent 
of $k$. So we have obtained a collection of ideals of every possible depth from 0 to $n-1$ such that the tropical variety is always the same.

The connection of depth $(S / I)$ with $\mathrm{gT}(I)$ is established by the following proposition, taken as a reformulation of [Herzog and Srinivasan 1998, Lemma 3.1] and relying on [Eliahou and Kervaire 1990]. Since a generic initial ideal $J$ is a monomial ideal, there exists a system of monomial generators of $J$. The unique smallest system of monomial generators with respect to inclusion will be called a minimal system of generators, and its elements are minimal generators of $J$.

Proposition 4.3. Let $I \subset S=K\left[x_{1}, \ldots, x_{n}\right]$ be a graded ideal with $\operatorname{dim}(S / I)=m$, and $\succ$ be any term order with $x_{1} \succ \cdots \succ x_{n}$. Let $\operatorname{depth}\left(S / \operatorname{gin}_{\succ}(I)\right)=t$. Then:

- Every minimal generator of $\operatorname{gin}_{\succ}(I)$ is divisible by one of $x_{1}, \ldots, x_{n-m}$.

- $x_{n-m}^{d}$ is one of the minimal generators of $\operatorname{gin}_{\succ}(I)$ for some $d \in \mathbb{N}$.

- The minimal generators of $\operatorname{gin}_{\succ}(I)$ are elements of $K\left[x_{1}, \ldots, x_{n-t}\right]$.

- There exists a minimal generator of $\operatorname{gin}_{\succ}(I)$ that is divisible by $x_{n-t}$.

In particular, if $\succ$ is the reverse lexicographic order, these statements are true for $t=\operatorname{depth}(S / I)$, since then $\operatorname{depth}(S / I)=\operatorname{depth}\left(S / \operatorname{gin}_{\succ}(I)\right)$.

Recall that by [Römer and Schmitz 2009, Corollary 7.4], the condition for $\omega \in$ $\mathbb{R}^{n}$ to be in $\mathrm{gT}(I)$ is that the minimum of its coordinates be attained at least $n-m+1$ times. So Proposition 4.3 already shows that the cases where $\operatorname{depth}(S / I)=m$ and $\operatorname{depth}(S / I)=m-1$ are special. We use the following standard definition.

Definition 4.4. Let $I \subset S=K\left[x_{1}, \ldots, x_{n}\right]$ be a graded ideal. If $\operatorname{depth}(S / I)=$ $\operatorname{dim}(S / I)$, then $S / I$ will be called Cohen-Macaulay. If $\operatorname{depth}(S / I)=\operatorname{dim}(S / I)-$ 1 , then $S / I$ is called almost-Cohen-Macaulay. In what follows, we say I is CohenMacaulay or almost-Cohen-Macaulay if $S / I$ has the corresponding property.

In this case, the refinement $\succ_{\omega}$ of every $\omega \in \mathrm{gT}(I)$ with respect to an appropriate reverse lexicographic order $\succ$ yields the same generic initial ideal as with respect to $\succ$. In the following statement, the set $U$ denotes the Zariski-open subset of $\mathrm{GL}_{n}(K)$ as defined in (2-1).

Lemma 4.5. Let $I \subset S=K\left[x_{1}, \ldots, x_{n}\right]$ be a graded Cohen-Macaulay or almostCohen-Macaulay ideal, $\succ$ be the reverse lexicographic order, and $\omega \in W_{n}^{m} \subset$ $\mathbb{R}^{n}$ with $\omega_{1}=\omega_{2}=\cdots=\omega_{n-m+1} \leq \omega_{n-m+2}, \ldots, \omega_{n}$. Moreover, let $\succ_{\omega}$ be the refinement of $\omega$ with respect to $\succ$. Then the reduced Gröbner bases of $g(I)$ with respect to $\succ$ and $\succ_{\omega}$ are the same for $g \in U$. In particular, $\operatorname{gin}_{\succ_{\omega}}(I)=\operatorname{gin}_{\succ}(I)$.

Proof. Since for a given degree $t$ any term containing none of $x_{n-m+2}, \ldots, x_{n}$ is smaller than any term divisible by one of them with respect to $\succ_{\omega}$, the term orders $\succ$ and $\succ_{\omega}$ coincide up to the term $x_{n-m+1}^{t}$. By Proposition 4.3, the minimal generators 
of $\operatorname{gin}_{\succ}(I)$ are monomials in $K\left[x_{1}, \ldots, x_{n-m+1}\right]$. Then for $g \in U$, the leading terms of the reduced Gröbner basis $\mathscr{G}(g)$ of $g(I)$ are terms in $K\left[x_{1}, \ldots, x_{n-m+1}\right]$. Since the leading terms of two elements of $\mathscr{G}(g)$ are the same with respect to $\succ$ and $\succ_{\omega}$, every S-pair with respect to $\succ_{\omega}$ is the same as with respect to $\succ$. As $\mathscr{G}(g)$ is a Gröbner basis with respect to $\succ$, every such S-pair reduces to 0 . So the set $\mathscr{G}(g)$ is a Gröbner basis with respect to $\succ_{\omega}$ as well. Hence, $\operatorname{gin}_{\succ_{\omega}}(I)=\operatorname{gin}_{\succ}(I)$.

We can now formulate the reverse statement of Proposition 3.7 for CohenMacaulay and almost-Cohen-Macaulay ideals.

Proposition 4.6. Let $I \subset S=K\left[x_{1}, \ldots, x_{n}\right]$ be a graded Cohen-Macaulay or almost-Cohen-Macaulay ideal with $\operatorname{dim}(S / I)=m$. Then for every relatively open cone $\stackrel{\circ}{C}_{A} \subset \mathcal{W}_{n}^{m}$ there exists a relatively open cone $\stackrel{\circ}{\mathrm{C}}$ of $\mathrm{gT}(I)$ with $\stackrel{\circ}{C}_{A} \subset \stackrel{\circ}{\mathrm{C}}$.

Proof. Let $A \subset\{1, \ldots, n\}$ with $|A| \geq n-m+1$, so $\stackrel{\circ}{C}_{A}$ is an open cone of $\mathscr{W}_{n}^{m}$. We need to show that for $\omega, \omega^{\prime} \in \stackrel{\circ}{C}_{A}$, we have $\operatorname{in}_{\omega}(g(I))=\operatorname{in}_{\omega^{\prime}}(g(I))$ for every $g \in U$. Without loss of generality, we may assume $\{1, \ldots, n-m+1\} \subset A$. Let $\succ$ denote the reverse lexicographic order. By Lemma 4.5, the reduced Gröbner basis $\mathscr{G}(g)=\left\{h_{1}(g), \ldots, h_{s}(g)\right\}$ of $g(I)$ with respect to $\succ$ is also a reduced Gröbner basis with respect to $\succ_{\omega}$ and $\succ_{\omega^{\prime}}$ for $g \in U$. So $\left\{\operatorname{in}_{\omega}\left(h_{1}(g)\right), \ldots, \operatorname{in}_{\omega}\left(h_{s}(g)\right)\right\}$ and $\left\{\operatorname{in}_{\omega^{\prime}}\left(h_{1}(g)\right), \ldots, \operatorname{in}_{\omega^{\prime}}\left(h_{s}(g)\right)\right\}$ are Gröbner bases of $\operatorname{in}_{\omega}(g(I))$ and $\operatorname{in}_{\omega^{\prime}}(g(I))$. However, all the leading terms of the $h_{i}(g)$ are elements of $K\left[x_{1}, \ldots, x_{n-m+1}\right]$. Hence, $\operatorname{in}_{\omega}\left(h_{i}(g)\right)$ and $\operatorname{in}_{\omega^{\prime}}\left(h_{i}(g)\right)$ exactly consist of those terms of $h_{i}(g)$ that contain only variables $x_{j}$ for which $\omega_{j}$ and $\omega_{j}^{\prime}$ respectively are minimal. But these variables are the same for $\omega$ and $\omega^{\prime}$ by assumption, so we obtain $\operatorname{in}_{\omega}(g(I))=$ $\operatorname{in}_{\omega^{\prime}}(g(I))$. This shows that all $\omega \in \stackrel{\circ}{C}_{A}$ are contained in the same open cone $\stackrel{\circ}{C}$ of $T(g(I))=\mathrm{gT}(I)$ for $g \in U$.

In the case of a Cohen-Macaulay or almost-Cohen-Macaulay ideal $I$ such that $\operatorname{dim}(S / I)=m$, the generic tropical variety is equal to $W_{n}^{m}$ as a fan. This generalizes the result [Römer and Schmitz 2009, Corollary 7.4] for this class of ideals.

Corollary 4.7. Let $I \subset S=K\left[x_{1}, \ldots, x_{n}\right]$ be a graded Cohen-Macaulay or almostCohen-Macaulay ideal with $\operatorname{dim}(S / I)=m$. Then $\mathrm{gT}(I)=W_{n}^{m}$ as a fan.

Proof. Let $\stackrel{\circ}{C}$ be a relatively open cone of $\mathrm{gT}(I)$. By Corollary 3.8, there exists a cone $D$ of $\mathcal{W}_{n}^{m}$ such that $\stackrel{\circ}{C} \subset \stackrel{\circ}{D}$. On the other hand, by Proposition 4.6 there exists a cone $E$ of $\mathrm{gT}(I)$ with $\stackrel{\circ}{D} \subset \stackrel{\circ}{E}$. But then $\stackrel{\circ}{C} \subset \stackrel{\circ}{E}$ are two cones of $\mathrm{gT}(I)$ with $\stackrel{\circ}{\mathrm{C}} \cap \stackrel{\circ}{E} \neq \varnothing$. This implies $\stackrel{\circ}{\mathrm{C}}=\stackrel{\circ}{E}$ and thus $\stackrel{\circ}{\mathrm{C}}=\stackrel{\circ}{D}$. This shows that every maximal cone of $\operatorname{gT}(I)$ is equal to some maximal cone of $W_{n}^{m}$. By the same argument, it follows that every maximal cone from $\mathcal{W}_{n}^{m}$ is equal to some maximal cone of $\mathrm{gT}(I)$, so the two fans are the same.

To show that Corollary 4.7 is wrong for every ideal that is not Cohen-Macaulay or almost-Cohen-Macaulay, we need the following auxiliary result. 
Lemma 4.8. Let $c \in \mathbb{N}$ and $\omega \in \mathbb{R}^{n}$ be such that $0=\omega_{1}=\cdots=\omega_{n-m+1}$ and $c \omega_{i}<\omega_{i+1}$ for $i=n-m+1, \ldots, n-1$. Let $\succ$ be the reverse lexicographic order. Then $\succ$ and $\succ_{\omega}$ are the same term orders for the monomials of any degree up to $c$.

Proof. Let $t \leq c$ and $x^{\nu}, x^{\mu}$ be two monomials of degree $t$. We write $x^{v}=y_{1} z_{1}$ and $x^{\mu}=y_{2} z_{2}$, where $y_{1}, y_{2} \in K\left[x_{1}, \ldots, x_{n-m+1}\right]$ and $z_{1}, z_{2} \in K\left[x_{n-m+2}, \ldots, x_{n}\right]$. If $z_{1}=z_{2}$, it is clear from the definition that $x^{\nu} \succ x^{\mu}$ if and only if $x^{\nu} \succ_{\omega} x^{\mu}$. Otherwise, let $k \geq n-m+2$ be the largest index such that $\nu_{k} \neq \mu_{k}$. Without loss of generality, we may assume that no variable $x_{j}$ divides $x^{\nu}$ or $x^{\mu}$ for $j>k$ and that $v_{k}<\mu_{k}$, so $x^{\nu} \succ x^{\mu}$. For the $\omega$-weight of $x^{\nu}$ and $x^{\mu}$ we obtain the upper bound

$$
\mathrm{wt}_{\omega}\left(x^{\nu}\right) \leq \mathrm{wt}_{\omega}\left(x_{k-1}^{t-v_{k}} x_{k}^{v_{k}}\right)=\omega_{k-1}\left(t-v_{k}\right)+\omega_{k} v_{k}
$$

and the lower bound

$$
\mathrm{wt}_{\omega}\left(x^{\mu}\right) \geq \mathrm{wt}_{\omega}\left(x_{1}^{t-\mu_{k}} x_{k}^{\mu_{k}}\right)=\omega_{k} \mu_{k} .
$$

So it is enough to show that $\omega_{k-1}\left(t-v_{k}\right)+\omega_{k} v_{k}<\omega_{k} \mu_{k}$. We have

$$
\begin{aligned}
c\left(\omega_{k} \mu_{k}-\left(\omega_{k-1}\left(t-v_{k}\right)+\omega_{k} v_{k}\right)\right) & =c \omega_{k}\left(\mu_{k}-v_{k}\right)-c \omega_{k-1}\left(t-v_{k}\right) \\
& >c \omega_{k}\left(\mu_{k}-v_{k}\right)-\omega_{k}\left(t-v_{k}\right) \\
& =\omega_{k}\left(c\left(\mu_{k}-v_{k}\right)-\left(t-v_{k}\right)\right) \geq 0 .
\end{aligned}
$$

The last inequality is true, since $t-v_{k} \leq c$ and $c\left(\mu_{k}-v_{k}\right)>c$, as we know $\mu_{k}>v_{k}$. It follows that $\mathrm{wt}_{\omega}\left(x^{\nu}\right)<\mathrm{wt}_{\omega}\left(x^{\mu}\right)$, so $x^{\nu} \succ_{\omega} x^{\mu}$. Hence, $\succ$ and $\succ_{\omega}$ coincide up to degree $c$.

We can now completely characterize when $\mathrm{gT}(I)$ is equal to a skeleton of the generic tropical fan for ideals of $\operatorname{depth}(S / I)>0$. If $\operatorname{dim}(S / I)=0$, we know that $\mathrm{gT}(I)$ is empty, since every graded ideal with $\operatorname{dim}(S / I)=0$ contains a monomial. In the cases $\operatorname{dim}(S / I)=1$ and $\operatorname{dim}(S / I)=2$, the fan $\mathrm{gT}(I)$ is equal to $W_{n}^{1}$ and $W_{n}^{2}$ respectively by [Römer and Schmitz 2009, Examples 8.3 and 8.4]. Note that in these cases, every ideal of $\operatorname{depth}(S / I)>0$ is Cohen-Macaulay or almost CohenMacaulay. For ideals with arbitrary dimension $\operatorname{dim}(S / I)>0$, we have:

Theorem 4.9. Let $I \subset S=K\left[x_{1}, \ldots, x_{n}\right]$ be a graded ideal with $\operatorname{dim}(S / I)=m>0$ and $\operatorname{depth}(S / I)>0$. Then $S / I$ is Cohen-Macaulay or almost-Cohen-Macaulay if and only if $\mathrm{gT}(I)=W_{n}^{m}$ as a fan.

Proof. We show that if $t=\operatorname{depth}(S / I)<m-1$, then $\mathrm{gT}(I) \neq W_{n}^{m}$ as a fan. For this, let $\succ$ be the reverse lexicographic order with $x_{1} \succ \cdots \succ x_{n-t} \succ x_{n-t+1} \succ \cdots \succ x_{n}$ and $\succ^{\prime}$ be the reverse lexicographic order with $x_{1} \succ^{\prime} \cdots \succ^{\prime} x_{n-t+1} \succ^{\prime} x_{n-t} \succ^{\prime} \cdots \succ^{\prime} x_{n}$. Let $c$ be the maximal degree of the minimal generators of $\operatorname{gin}_{\succ}(I)$ and $\operatorname{gin}_{\succ^{\prime}}(I)$. For the purpose of this proof, for $a, b \in \mathbb{R}_{+}$we write $a \ll b$ if $a c<b$. 
Choose $\omega, v \in \mathbb{R}^{n}$ such that $0=\omega_{1}=\cdots=\omega_{n-m+1} \ll \omega_{n-m+2} \ll \cdots \ll \omega_{n}$ and

$$
0=v_{1}=\cdots=v_{n-m+1} \ll v_{n-m+2} \ll \cdots \ll v_{n-t+1} \ll v_{n-t} \ll \cdots \ll v_{n} .
$$

By Lemma 4.8, we know that $\succ$ and $\succ_{\omega}$ are the same term orders up to degree $c$. Since $\operatorname{gin}_{\succ}(I)$ is generated by monomials of degree at most $c$, for a fixed $g \in U$ (as defined in (2-1)), all elements of the reduced Gröbner basis $\mathscr{G}$ of $g(I)$ with respect to $\succ$ have degree at most $c$. The leading term of every element of $\mathscr{G}$ is the same with respect to $\succ$ and $\succ_{\omega}$. Thus every S-pair of elements of $\mathscr{G}$ reduces to zero with respect to $\succ_{\omega}$ as well. So $\mathscr{G}$ is also a Gröbner basis of $\operatorname{gin}_{\succ_{\omega}}(I)$. This implies $\operatorname{gin}_{\succ \omega}(I)=\operatorname{gin}_{\succ}(I)$.

By Lemma 4.8 and the same argument as before, we can show that $\operatorname{gin}_{\succ_{v}}(I)=$ $\operatorname{gin}_{\succ^{\prime}}(I)$. Since $\operatorname{depth}\left(S / \operatorname{gin}_{\succ}(I)\right)=\operatorname{depth}\left(S / \operatorname{gin}_{\succ^{\prime}}(I)\right)=t$, we know that $x_{n-t}$ divides one of the minimal generators of $\operatorname{gin}_{\succ}(I)$ but does not divide one of the minimal generators of $\operatorname{gin}_{\succ^{\prime}}(I)$ by Proposition 4.3. So $\operatorname{gin}_{\succ_{\omega}}(I)=\operatorname{gin}_{\succ}(I) \neq$ $\operatorname{gin}_{\succ^{\prime}}(I)=\operatorname{gin}_{\succ_{v}}(I)$, and it follows that $\operatorname{in}_{\omega}(g(I)) \neq \operatorname{in}_{v}(g(I))$ for $g \in U$. Hence, $\omega$ and $v$ are not in the same relatively open cone of $\mathrm{gT}(I)$, but they are in the same relatively open cone of $\mathcal{W}_{n}^{m}$. This implies $\mathrm{gT}(I) \neq \mathcal{W}_{n}^{m}$ as a fan.

The converse of this statement, that $\operatorname{depth}(S / I) \geq m-1 \operatorname{implies} \operatorname{gT}(I)=\mathscr{W}_{n}^{m}$ as a fan, has already been proved in Corollary 4.7.

In particular, this theorem gives a negative answer to the question posed in the introduction of [Römer and Schmitz 2009] of whether the generic tropical variety of $I$ as a fan only depends on the dimension of $S / I$. If $\operatorname{depth}(S / I)=0$, it is not possible to obtain a statement like Theorem 4.9; see Remark 5.12.

\section{Generic tropical varieties and depth}

In this section we will consider a certain class of ideals $I$ such that $\operatorname{dim}(S / I)-1>$ $\operatorname{depth}(S / I)>0$ for which we can recover the depth from the generic tropical varieties. These ideals have the property that the rings $S / \operatorname{gin}_{\succ}(I)$ for all generic initial ideals of $I$ have the same depth as $S / I$ itself. This makes it possible to use Proposition 4.3 on all of these. We express this property by considering the generic depth of $S / I$.

Definition 5.1. For a graded ideal $I \subset S=K\left[x_{1}, \ldots, x_{n}\right]$, we call

$$
\operatorname{gdepth}(S / I)=\min \left\{\operatorname{depth}\left(S / \operatorname{gin}_{\succ}(I)\right)\right\},
$$

where the minimum is taken over all possible generic initial ideals of $I$, the generic depth of $S / I$. If $\operatorname{depth}(S / I)=\operatorname{gdepth}(S / I)$, then $I$ is called a maximal-gdepth ideal.

Note that since $\operatorname{depth}\left(S / \operatorname{gin}_{\succ}(I)\right) \leq \operatorname{depth}(S / I)$ for any generic initial ideal of $I$, the ideal $I$ is a maximal-gdepth ideal if and only if $\operatorname{depth}(S / I)=\operatorname{depth}\left(S / \operatorname{gin}_{\succ}(I)\right)$ 
for every generic initial ideal of $I$. A trivial example of a maximal-gdepth ideal is a principal ideal like $I=\left(x_{1}^{d}+\cdots+x_{n}^{d}\right)$ in $S=K\left[x_{1}, \ldots, x_{n}\right]$ for an integer $d \geq 1$, because every gin is also a principal ideal generated by a polynomial of degree $d$. Next we describe two interesting classes of maximal-gdepth ideals.

Example 5.2. The first example is the class of strongly stable ideals $I$. These are by definition monomial ideals, so the tropical variety $T(I)$ is of course empty. We are, however, interested in the generic tropical variety $\mathrm{gT}(I)$. This fan is not empty if $\operatorname{dim}(S / I)>0$, and it contains algebraic information on $I$, as we show.

Recall that a monomial ideal $I \subset K\left[x_{1}, \ldots, x_{n}\right]$ is called strongly stable with respect to some ordering $x_{i_{1}}>\cdots>x_{i_{n}}$ of the variables $x_{1}, \ldots, x_{n}$ if for every monomial $u \in I$ we also have $x_{i_{j}} u x_{i_{k}}^{-1} \in I$ for every $x_{i_{k}}$ that divides $u$ and every $j<k$. Every ideal $\operatorname{gin}_{\succ}(I)$ is a strongly stable ideal with respect to the ordering of the variables given by $\succ$, since $\operatorname{char}(K)=0$. Moreover, if $I$ is strongly stable with respect to $x_{i_{1}}>\cdots>x_{i_{n}}$ and $\succ$ is a term order with $x_{i_{1}} \succ \cdots \succ x_{i_{n}}$, then $\operatorname{gin}_{\succ}(I)=I$. We now explain that strongly stable ideals are maximal-gdepth ideals.

Let $I \subset K\left[x_{1}, \ldots, x_{n}\right]$ be a graded strongly stable ideal with respect to $x_{1}>\cdots>$ $x_{n}$. Let $\succ$ be any term order with $x_{i_{1}} \succ \cdots \succ x_{i_{n}}$ for $\left\{i_{1}, \ldots, i_{n}\right\}=\{1, \ldots, n\}$. In addition, let $\succ^{\prime}$ be the reverse lexicographic order with $x_{i_{1}} \succ^{\prime} \cdots \succ^{\prime} x_{i_{n}}$. Consider the image of $I$ under the $K$-algebra isomorphism $\phi$ that maps $x_{j}$ to $x_{i_{j}}$. Then $\phi(I)$ is a strongly stable ideal with respect to term orders with $x_{i_{1}} \succ \cdots \succ x_{i_{n}}$, so in particular with respect to $\succ$ and to $\succ^{\prime}$. So we know that $\operatorname{gin}_{\succ}(\phi(I))=\operatorname{gin}_{\succ^{\prime}}(\phi(I))=\phi(I)$. Let $\varnothing \neq U_{1} \subset \mathrm{GL}_{n}(K)$ be Zariski-open such that $\operatorname{in}_{\succ}(g(I))=\operatorname{gin}_{\succ}(I)$ for every $g \in U_{1}$, and $\varnothing \neq U_{2} \subset \mathrm{GL}_{n}(K)$ Zariski-open such that $\operatorname{in}_{\succ}(h(\phi(I)))=\operatorname{gin}_{\succ}(\phi(I))$ for every $h \in U_{2}$. Note that also the set $\varnothing \neq U_{2}^{\prime}=\left\{h \circ \phi \in \mathrm{GL}_{n}(K): h \in U_{2}\right\}$ is Zariski-open, as it can be defined by the polynomials obtained by permuting the polynomials defining $U_{2}$ according to $\phi$. Hence, $U_{1} \cap U_{2}^{\prime} \neq \varnothing$. For $k \in U_{1} \cap U_{2}^{\prime}$ we have $\operatorname{in}_{\succ}(k(I))=\operatorname{gin}_{\succ}(I)=\operatorname{gin}_{\succ}(\phi(I))$. In addition, $\operatorname{gin}_{\succ^{\prime}}(I)=\operatorname{gin}_{\succ^{\prime}}(\phi(I))$ by the same argument. Hence, $\operatorname{gin}_{\succ}(I)=\operatorname{gin}_{\succ^{\prime}}(I)$. Since for any reverse lexicographic order $\succ$ we have $\operatorname{depth}\left(S / \operatorname{gin}_{\succ}(I)\right)=\operatorname{depth}(S / I)$, this implies that strongly stable ideals are maximal-gdepth ideals.

A concrete example for such an ideal is $\left(x_{1}^{2}, x_{1} x_{2}, x_{1} x_{3}, x_{1} x_{4}\right) \subseteq K\left[x_{1}, \ldots, x_{5}\right]$. Applying a $K$-algebra automorphism in $\mathrm{GL}_{n}(K)$ provides examples of maximalgdepth ideals that are not monomial ideals.

Example 5.3. The second example class is the class of ideals such that $I$ and every $\operatorname{gin}_{\succ}(I)$ is generated by polynomials of the same degree. We can see that these ideals are also maximal-gdepth as follows. Let $S=K\left[x_{1}, \ldots, x_{n}\right]$ with the standard $\mathbb{Z}$-grading and note that $S / I$ is a graded $S$-module. We denote by $\beta_{i, j}$ the graded Betti number $\beta_{i, j}=\beta_{i, j}(S / I)=\operatorname{dim}_{K}\left(\operatorname{Tor}_{i}^{S}(S / I, K)\right)_{j}$. For $d \in \mathbb{N}$, let $S(-d)$ be the graded module $S$ with the grading given by $S(-d)_{j}=S_{j-d}$. Recall 
that for some $d \in \mathbb{N}$, we say that $I$ has a $d$-linear resolution if and only if $\beta_{i, i+j}=0$ for $j \neq d-1, i \geq 1$. This is equivalent to the fact that the minimal graded free resolution of $S / I$ has the form

$$
0 \rightarrow S(-d-p+1)^{\beta_{p}} \rightarrow \cdots \rightarrow S(-d-1)^{\beta_{2}} \rightarrow S(-d)^{\beta_{1}} \rightarrow S \rightarrow S / I \rightarrow 0,
$$

where $\beta_{i}=\beta_{i, i+d}$ is the $i$-th total Betti number and $p$ is the projective dimension $p=\operatorname{projdim}(S / I)$ of $S / I$. Let $I \subset S$ be a graded ideal such that $I$ and $\operatorname{gin}_{\succ}(I)$ are generated by polynomials of degree $d$ for every term order $\succ$. Let $J=\operatorname{gin}_{\succ}(I)$ be a given generic initial ideal if $I$. We now show that $\operatorname{depth}(S / I)=\operatorname{depth}(S / J)$. As $J$ is strongly stable and generated in one degree, the minimal graded free resolution (as constructed in [Eliahou and Kervaire 1990]) is linear. Since $\beta_{i, j}(S / I) \leq$ $\beta_{i, j}(S / J)$ for every $i, j$ (see for example [Bruns and Conca 2004, Proposition 3.3]), this implies that $S / I$ has a linear resolution as well. So $I$ and $J$ have linear resolutions, which in turn means that their total Betti numbers depend only on the Hilbert series of $S / I$ and $S / J$ respectively. But $H_{S / I}(t)=H_{S / J}(t)$, so the Betti numbers and in particular the projective dimensions of $S / I$ and $S / J$ are the same. By the Auslander-Buchsbaum formula $\operatorname{depth}(S / I)+\operatorname{projdim}(S / I)=n$, it follows that $\operatorname{depth}(S / I)=\operatorname{depth}(S / J)$. This is true for every generic initial ideal of $I$. Hence, $I$ is a maximal-gdepth ideal.

Recall that the Castelnuovo-Mumford regularity $\operatorname{reg}(S / I)$ of $S / I$ is defined to be the maximal $j \in \mathbb{Z}$ such that $\beta_{i, i+j} \neq 0$ for some $i \geq 0$. It is well-known that the ideal $I_{\geq t}$ (generated by all homogeneous components $I_{s}$ of $I$ with $s \geq t$ ) has a linear resolution if $t \geq \operatorname{reg}(S / I)+1$; see [Eisenbud and Goto 1984]. Observe further that by the construction of gin, we know $\operatorname{gin}_{\succ}(I)_{\geq t}=\operatorname{gin}_{\succ}\left(I_{\geq t}\right)$. Since there exist only finitely many different generic initial ideals for $I$, we can find a $t$ such that all $\operatorname{gin}_{\succ}\left(I_{\geq t}\right)$ have a linear resolution. In particular, they are generated in degree $t$. Then also $I_{\geq t}$ is generated in degree $t$ (and has a linear resolution). So every high truncation $I_{\geq t}$ of an arbitrary graded ideal $I$ is a maximal-gdepth ideal. Unfortunately we cannot decide in general which $t$ one has to take.

In this section we give a structural result on generic tropical varieties of maximalgdepth ideals. We will see that these as fans are closely related to the following refinement of $\mathcal{W}_{n}^{m}$.

Definition 5.4. Let $\mathscr{W}_{n}^{m}$ be the $m$-skeleton of the standard tropical fan in $\mathbb{R}^{n}$, and let $0<t<m-1$. The refinement of $\mathcal{W}_{n}^{m}$ containing all open cones

$$
\left\{\omega \in \mathbb{R}^{n}: \omega_{i_{1}}=\cdots=\omega_{i_{n-m+1}}<\omega_{i_{n-m+2}}, \ldots, \omega_{i_{n-t}}<\omega_{i_{n-t+1}}, \ldots, \omega_{i_{n}}\right\}
$$

for any permutation $\left(i_{1}, \ldots, i_{n}\right)$ of $\{1, \ldots, n\}$ as maximal open cones will be called the $t$-refinement of $\mathcal{W}_{n}^{m}$ and denoted by $\mathcal{W}_{n}^{m, t}$. 
Lemma 5.5. Let $I \subset S=K\left[x_{1}, \ldots, x_{n}\right]$ be a maximal-gdepth ideal such that $\operatorname{dim}(S / I)=m<n$ and $\operatorname{depth}(S / I)=t$ for some $0<t<m-1$. Let $\omega \in \stackrel{\circ}{C}$ for some maximal cone $C$ of $\mathrm{gT}(I)$ and let $\omega_{i_{1}} \leq \cdots \leq \omega_{i_{n}}$ for $\left\{i_{1}, \ldots, i_{n}\right\}=\{1, \ldots, n\}$. Then $\omega_{i_{n-t}}<\omega_{i_{n-t+1}}$.

Proof. Without loss of generality we have

$$
\omega_{1}=\cdots=\omega_{n-m+1}<\omega_{n-m+2} \leq \cdots \leq \omega_{n},
$$

since $\omega \in\left|W_{n}^{m}\right|$. Let us assume that $\omega_{n-t}=\omega_{n-t+1}$. For $\varepsilon>0$ we define $u^{\varepsilon} \in \mathbb{R}^{n}$ by

$$
u_{i}^{\varepsilon}= \begin{cases}\omega_{i}-\varepsilon & \text { for } i<n-t \\ \omega_{i} & \text { for } i=n-t \\ \omega_{i}+\varepsilon & \text { for } i=n-t+1 \\ \omega_{i}+2 \varepsilon & \text { for } i>n-t+1\end{cases}
$$

In the same way we define $v_{i}^{\varepsilon} \in \mathbb{R}^{n}$ by

$$
v_{i}^{\varepsilon}= \begin{cases}\omega_{i}-\varepsilon & \text { for } i<n-t \\ \omega_{i} & \text { for } i=n-t+1 \\ \omega_{i}+\varepsilon & \text { for } i=n-t \\ \omega_{i}+2 \varepsilon & \text { for } i>n-t+1\end{cases}
$$

Note that $u^{\varepsilon}$ and $v^{\varepsilon}$ are contained in $\operatorname{gT}(I)$ for every choice of $\varepsilon$. Let $\succ$ be any term order. Then $x_{i} \succ_{u^{\varepsilon}} x_{n-t} \succ_{u^{\varepsilon}} x_{n-t+1} \succ_{u^{\varepsilon}} x_{j}$ and $x_{i} \succ_{v^{\varepsilon}} x_{n-t+1} \succ_{v^{\varepsilon}} x_{n-t} \succ_{v^{\varepsilon}} x_{j}$ for $i<n-t, j>n-t+1$. Since

$$
\operatorname{depth}(S / I)=\operatorname{depth}\left(S / \operatorname{gin}_{\succ_{u^{\varepsilon}}}(I)\right)=\operatorname{depth}\left(S / \operatorname{gin}_{\succ_{v^{\varepsilon}}}(I)\right),
$$

by Proposition 4.3 the monomial ideal $\operatorname{gin}_{\succ_{u}^{\varepsilon}}(I)$ contains a minimal monomial generator divisible by $x_{n-t}$, but none that is divisible by $x_{n-t+1}$. On the other hand, $\operatorname{gin}_{\succ_{v^{\varepsilon}}}(I)$ contains a minimal generator divisible by $x_{n-t+1}$, but none that is divisible by $x_{n-t}$. Hence, the reduced Gröbner bases of $\operatorname{in}_{u^{\varepsilon}}(g(I))$ and $\operatorname{in}_{v^{\varepsilon}}(g(I))$ are different with respect to the same term order $\succ$. Since the reduced Gröbner basis of an ideal is unique with respect to a given term order, this implies $\operatorname{in}_{u^{\varepsilon}}(g(I)) \neq$ $\operatorname{in}_{v^{\varepsilon}}(g(I))$, so $u^{\varepsilon}$ and $v^{\varepsilon}$ are in different cones of $\mathrm{gT}(I)$. So in every neighborhood of $\omega$ in $\mathrm{gT}(I)$, there are elements that are in different cones of $\mathrm{gT}(I)$. This is a contradiction to the fact that $\omega \in \stackrel{\circ}{C}$ and $C$ is maximal.

We can now show that for maximal-gdepth ideals, gT $(I)$ refines $\mathcal{W}_{n}^{m, t}$ as a fan.

Proposition 5.6. Let $I \subset S=K\left[x_{1}, \ldots, x_{n}\right]$ be a maximal-gdepth ideal with $\operatorname{dim}(S / I)=m<n$ and $0<\operatorname{depth}(S / I)=t<m-1$. Let $C$ be a maximal cone of $\mathrm{gT}(I)$. Then there exists a cone $D \subset \mathcal{W}_{n}^{m, t}$ such that $\stackrel{\circ}{C} \subset \stackrel{\circ}{D}$. 
Proof. By Lemma 5.5, we know that $\stackrel{\circ}{C}$ does not intersect the $(m-1)$-skeleton $X$ of $\mathscr{W}_{n}^{m, t}$. So $\stackrel{\circ}{C}$ must be contained in the union $\mathscr{W}_{n}^{m, t} \backslash X$ of the open maximal cones of $W_{n}^{m, t}$. Since it is convex, $\stackrel{\circ}{C}$ is connected and thus contained in one connected component of $\mathcal{W}_{n}^{m, t} \backslash X$. But the connected components of $\mathcal{W}_{n}^{m, t} \backslash X$ are the open maximal cones themselves, so $\stackrel{\circ}{C}$ must be contained in some maximal open cone $\stackrel{\circ}{D}$ of $\mathcal{W}_{n}^{m, t}$.

This shows that the fan $\mathrm{gT}(I)$ is always finer than the fan $\mathcal{W}_{n}^{m, t}$ for maximalgdepth ideals. We can now give a complementary result by showing that every maximal cone contains a $t$-dimensional orthant of $\mathbb{R}^{n}$. For this we will need the following basic observation from Gröbner basis theory.

Lemma 5.7. Let $I \subset\left[x_{1}, \ldots, x_{n}\right]$ be a graded ideal and let $\omega, \omega^{\prime} \in \mathbb{R}^{n}$. Let $\succ$ be a term order and $\varphi$ be the reduced Gröbner basis of I with respect to $\succ_{\omega}$. If $\operatorname{in}_{\omega}(f)=\operatorname{in}_{\omega^{\prime}}(f)$ for every $f \in G$, then $\operatorname{in}_{\omega}(I)=\operatorname{in}_{\omega^{\prime}}(I)$.

Proof. Since $\left\{\operatorname{in}_{\omega}(f): f \in \mathscr{G}\right\}$ is a reduced Gröbner basis for $\operatorname{in}_{\omega}(I)$ with respect to $\succ$ (see for example [Maclagan and Thomas 2007, Lemma 2.4.2]), it follows that $\operatorname{in}_{\omega}(I)=\left(\operatorname{in}_{\omega^{\prime}}(f): f \in \mathscr{G}\right) \subset \operatorname{in}_{\omega^{\prime}}(I)$. This implies that $\operatorname{in}_{\succ_{\omega}}(I) \subset \operatorname{in}_{\succ_{\omega^{\prime}}}(I)$. As there cannot be a proper inclusion of two initial ideals (see [Maclagan and Thomas 2007, Corollary 2.2.3]), this means $\operatorname{in}_{\succ_{\omega}}(I)=\operatorname{in}_{\succ_{\omega^{\prime}}}(I)$. Therefore we have $\operatorname{in}_{\omega}(I)=\operatorname{in}_{\omega^{\prime}}(I)$, because $\left\{\operatorname{in}_{\omega}(f): f \in \mathscr{G}\right\}$ is also a reduced Gröbner basis of $g(I)$ with respect to $\succ_{\omega^{\prime}}$.

Proposition 5.8. Let $I \subset S=K\left[x_{1}, \ldots, x_{n}\right]$ be a maximal-gdepth ideal with $\operatorname{dim}(S / I)=m$, let $0<\operatorname{depth}(S / I)=t<m-1$, and let $c$ be the maximal total degree of a minimal generator of a generic initial ideal of $I$. Let $\omega \in \mathrm{gT}(I)$ with

$$
0=\omega_{1}=\cdots=\omega_{n-m+1}<\omega_{n-m+2} \leq \cdots \leq \omega_{n-t}<\omega_{n-t+1}, \ldots, \omega_{n}
$$

such that $\omega_{n-t} c<\omega_{j}$ for $j>n-t$ and $\omega \in \stackrel{\circ}{C}$ for some maximal cone $C$ of $\mathrm{gT}(I)$. Then

$$
\omega+\operatorname{cone}\left(e_{n-t+1}, \ldots, e_{n}\right) \subset \stackrel{\circ}{C},
$$

where $e_{i}$ denotes the $i$-th standard basis vector of $\mathbb{R}^{n}$.

Proof. Let $\succ$ be any term order. Then for the refinement $\succ_{\omega}$ of $\omega$ by Proposition 4.3 , the generic initial ideal $\operatorname{gin}_{\succ_{\omega}}(I)$ is minimally generated in $K\left[x_{1}, \ldots, x_{n-t}\right]$, since $I$ is maximal-gdepth and $\operatorname{depth}\left(S / \operatorname{gin}_{\succ_{\omega}}(I)\right)=\operatorname{depth}(S / I)=t$. Hence, for $g \in U$ (where $U$ is defined as in (2-1)) there exists a reduced Gröbner basis $\mathscr{G}$ of $g(I)$ with respect to $\succ_{\omega}$ such that $\operatorname{in}_{\succ_{\omega}}(f) \in K\left[x_{1}, \ldots, x_{n-t}\right]$ for every $f \in \mathscr{G}$.

We show that $\operatorname{in}_{\omega^{\prime}}(f) \in K\left[x_{1}, \ldots, x_{n-t}\right]$ for every $\omega^{\prime} \in \omega+\operatorname{cone}\left(e_{n-t+1}, \ldots, e_{n}\right)$ and every $f \in \mathscr{G}$. To see this, we need to show that every term of $f$ that contains one of the $x_{n-t+1}, \ldots, x_{n}$ has larger $\omega^{\prime}$-weight than any term of $f$ in $K\left[x_{1}, \ldots, x_{n-t}\right]$. By the choice of $c$, the $\omega^{\prime}$-weight of a term of $f$ in $K\left[x_{1}, \ldots, x_{n-t}\right]$ is bounded 
from above by $c \omega_{n-t}$. But any of the $x_{n-t+1}, \ldots, x_{n}$ has weight strictly larger than $c \omega_{n-t}$. Since we already know that $f$ contains a term in $K\left[x_{1}, \ldots, x_{n-t}\right]$, we have $\operatorname{in}_{\omega^{\prime}}(f) \in K\left[x_{1}, \ldots, x_{n-t}\right]$. By the choice of $\omega^{\prime}$, all terms in $K\left[x_{1}, \ldots, x_{n-t}\right]$ have the same $\omega$-weight and $\omega^{\prime}$-weight. So $\operatorname{in}_{\omega^{\prime}}(f)=\operatorname{in}_{\omega}(f)$ for $f \in \mathscr{G}$. Then by Lemma 5.7, it follows that $\operatorname{in}_{\omega}(g(I))=\operatorname{in}_{\omega^{\prime}}(g(I))$ for $g \in U$. Hence, $\omega^{\prime} \in \stackrel{C}{C}$ for every $\omega^{\prime} \in \omega+\operatorname{cone}\left(e_{n-t+1}, \ldots, e_{n}\right)$.

For maximal-gdepth ideals $I$, it is therefore possible to obtain depth $(S / I)$ from the generic tropical variety of $I$, as shown in the following theorem.

Theorem 5.9. Let $I \subset S=K\left[x_{1}, \ldots, x_{n}\right]$ be a maximal-gdepth ideal such that $\operatorname{dim}(S / I)=m$ and $0<\operatorname{depth}(S / I)<m-1$. Then

$$
\operatorname{depth}(S / I)=\min \left\{t \in \mathbb{N}: \operatorname{gT}(I) \text { refines } \mathcal{W}_{n}^{m, t}\right\} .
$$

Proof. Let $T=\operatorname{depth}(S / I)$. By Proposition 5.6, we already know that $\mathrm{gT}(I)$ refines $\mathcal{W}_{n}^{m, T}$ as a fan. On the other hand let $t<T$. Then we can choose $\omega \in \mathbb{R}^{n}$ such that

$$
\omega_{1}=\cdots=\omega_{n-m+1}<\omega_{n-m+2}, \ldots, \omega_{n-t}<\omega_{n-t+1}, \ldots, \omega_{n}
$$

with $\omega \in \stackrel{\circ}{ }$ for some maximal cone $C$ of $\operatorname{gT}(I)$ and $\omega_{n-t} c<\omega_{j}$ for $j>n-t$, where $c$ is chosen as in Proposition 5.8. Define $\omega_{i}^{\prime}=\omega_{i}$ for $i \neq n-t$ and choose $\omega_{n-t}^{\prime}>\omega_{n-t+1}$. Since $t<T$, by Proposition 5.8 we know that $\omega^{\prime} \in \stackrel{\circ}{\mathrm{C}}$ as well. But by definition of $\mathcal{W}_{n}^{m, t}$, we know that $\omega$ and $\omega^{\prime}$ are in different open cones of $\mathcal{W}_{n}^{m, t}$. So gT $(I)$ cannot refine $W_{n}^{m, t}$ as a fan.

Remark 5.10. Note that it is also possible to recover depth $(S / I)$ from $\mathrm{gT}(I)$ for arbitrary graded ideals $I \subset K\left[x_{1}, \ldots, x_{n}\right]$ with $\operatorname{dim}(S / I)=m$ and $0<\operatorname{depth}(S / I)<$ $m-1$ in the following way. Let $\operatorname{depth}(S / I)=t$ and let $\succ$ be the reverse lexicographic term order. Let $c$ be the maximal degree of a minimal generator of any generic initial ideal with respect to a reverse lexicographic term order. Choose $\omega \in \stackrel{\circ}{C}$ for some maximal cone $C$ of $\mathrm{gT}(I)$ as in Lemma 4.8. We now show that for this particular choice of $\omega$, we have

$$
\omega+\operatorname{cone}\left(e_{n-t+1}, \ldots, e_{n}\right) \subset \stackrel{\circ}{C},
$$

but

$$
\omega+\operatorname{cone}\left(e_{n-t}, \ldots, e_{n}\right) \not \subset \stackrel{\circ}{C} .
$$

Since $\succ$ and $\succ_{\omega}$ coincide up to degree $c$, this implies $\operatorname{gin}_{\succ_{\omega}}(I)=\operatorname{gin}_{\succ}(I)$. In $\operatorname{particular}, \operatorname{depth}\left(S / \operatorname{gin}_{\succ_{\omega}}(I)\right)=\operatorname{depth}(S / I)$. By the same proof as in Proposition 5.8, we obtain that $\omega+\operatorname{cone}\left(e_{n-t+1}, \ldots, e_{n}\right) \subset \stackrel{\circ}{\mathrm{C}}$.

Assume that $\omega+\operatorname{cone}\left(e_{n-t}, \ldots, e_{n}\right) \subset \stackrel{\circ}{C}$. Let $\succ^{\prime}$ be the reverse lexicographic term order with $x_{1} \succ^{\prime} \cdots \succ^{\prime} x_{n-t-1} \succ^{\prime} x_{n-t+1} \succ^{\prime} \cdots \succ^{\prime} x_{n} \succ^{\prime} x_{n-t}$. Then we define $\omega^{\prime} \in \mathbb{R}^{n}$ by $\omega_{i}^{\prime}=\omega_{i}$ for $i \neq n-t$ and $\omega_{n-t}^{\prime}>\omega_{n} c$. By assumption, we know that 
$\omega^{\prime} \in \stackrel{\circ}{C}$. Since $\succ_{\omega^{\prime}}$ and $\succ^{\prime}$ coincide up to degree $c$ by Lemma 4.8, we know that $\operatorname{gin}_{\succ_{\omega^{\prime}}}(I)=\operatorname{gin}_{\succ^{\prime}}(I)$. As in the proof of Theorem 4.9, we get that

$$
\operatorname{gin}_{\succ_{\omega}}(I)=\operatorname{gin}_{\succ}(I) \neq \operatorname{gin}_{\succ^{\prime}}(I)=\operatorname{gin}_{\succ_{\omega^{\prime}}}(I) .
$$

This implies $\operatorname{in}_{\omega}(g(I)) \neq \operatorname{in}_{\omega^{\prime}}(g(I))$ for $g \in U$, which is a contradiction to $\omega, \omega^{\prime} \in \stackrel{\circ}{\text {. }}$.

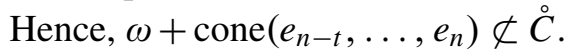

To obtain depth $(S / I)$ from $\operatorname{gT}(I)$, we can therefore determine $\omega$ as described above. Then we have

$$
\operatorname{depth}(S / I)=\min \left\{t: \omega+\operatorname{cone}\left(e_{n-t+1}, \ldots, e_{n}\right) \subset \stackrel{\circ}{C}\right\}
$$

for this particular choice of $\omega$.

As we saw in Proposition 5.6, the generic tropical variety of a maximal-gdepth ideal with $\operatorname{dim}(S / I)=m$ and $\operatorname{depth}(S / I)=t$ with $0<t<m-1$ always refines $W_{n}^{m, t}$. It is also true that any of the fans $\mathcal{W}_{n}^{m, t}$ is the generic tropical variety of some ideal, which we will see by focusing on a class of strongly stable ideals generated in degree 2.

Proposition 5.11. Let $0<m<n$ and $0<t<m-1$. The ideal

$$
I=\left(x_{1}, \ldots, x_{n-m-1}, x_{n-m}^{2}, x_{n-m} x_{n-m+1}, \ldots, x_{n-m} x_{n-t}\right) \subset S=K\left[x_{1}, \ldots, x_{n}\right]
$$

is a maximal-gdepth ideal with $\operatorname{dim}(S / I)=m, \operatorname{depth}(S / I)=t$ and $\operatorname{gT}(I)=W_{n}^{m, t}$ as a fan.

Proof. We first show that $\operatorname{dim}(S / I)=m$ and $\operatorname{depth}(S / I)=t$. Since $I$ is strongly stable with respect to the reverse lexicographic order $\succ$, we have $\operatorname{gin}_{\succ}(I)=I$. So $I$ has only one minimal prime by [Eisenbud 1995, Corollary 15.25], which is $\left(x_{1}, \ldots, x_{n-m}\right)$. Thus, $\operatorname{dim}(S / I)=m$. To see that $\operatorname{depth}(S / I)=t$, we note again that $\operatorname{gin}_{\succ}(I)=I$. By Proposition 4.3, it follows that $\operatorname{depth}(S / I)=n-(n-t)=t$. In particular, $I$ is a maximal-gdepth ideal (see Example 5.2).

By Proposition 5.6, we know that every maximal cone $\stackrel{\circ}{C}$ of $\operatorname{gT}(I)$ is contained in some maximal cone $\stackrel{\circ}{D}$ of ${ }^{q} W_{n}^{m, t}$. So it remains to show that for every $\omega, \omega^{\prime} \in \stackrel{\circ}{D}$ for some maximal cone $D$ of $W_{n}^{m, t}$, we have $\operatorname{in}_{\omega}(g(I))=\operatorname{in}_{\omega^{\prime}}(g(I))$ for $g \in U$. Let $D$ be the maximal cone of $\mathcal{W}_{n}^{m, t}$ given by

$$
\stackrel{\circ}{D}=\left\{\omega \in \mathbb{R}^{n}: \omega_{i_{1}}=\cdots=\omega_{i_{n-m+1}}<\omega_{i_{n-m+2}}, \ldots, \omega_{i_{n-t}}<\omega_{i_{n-t+1}}, \ldots, \omega_{i_{n}}\right\}
$$

for some permutation $\left(i_{1}, \ldots, i_{n}\right)$ of $\{1, \ldots, n\}$. Let $\omega \in \stackrel{D}{D}$ be fixed, $\succ_{\omega}$ be the refinement of $\omega$ with respect to the reverse lexicographic order $\succ$ with $x_{i_{n-m+2}} \succ$ $\cdots \succ x_{i_{n}} \succ x_{i_{1}} \succ \cdots \succ x_{i_{n-m+1}}$, and $\mathscr{G}$ be the reduced Gröbner basis of $g(I)$ with respect to $\succ_{\omega}$ for a fixed $g \in U$. Note that $x_{i_{1}} \succ_{\omega} \cdots \succ_{\omega} x_{i_{n-m+1}}$ and $x_{i_{k}} \succ_{\omega} x_{i_{j}}$ for $k \in\{n-m+2, \ldots, n-t\}, j \in\{n-t+1, \ldots, n\}$. Let $\left(q_{1}, \ldots, q_{n}\right)$ be the permutation 
on $\{1, \ldots, n\}$ such that $x_{q_{1}} \succ_{\omega} x_{q_{2}} \succ_{\omega} \cdots \succ_{\omega} x_{q_{n}}$. As in Example 5.2, we know $\operatorname{gin}_{\succ_{\omega}}(I)=\phi(I)$ for the $K$-algebra isomorphism $\phi$ induced by $\phi\left(x_{j}\right)=x_{q_{j}}$. So

$$
\operatorname{gin}_{\succ_{\omega}}(I)=\left(x_{i_{1}}, \ldots, x_{i_{n-m-1}}, x_{i_{n-m}}^{2}, x_{i_{n-m}} x_{i_{n-m+1}}, \ldots, x_{i_{n-m}} x_{i_{n-t}}\right) .
$$

Hence, $\operatorname{in}_{\succ \omega}(f)=x_{i_{j}}$ for some $j \in\{1, \ldots, n-m-1\}$ or $\operatorname{in}_{\succ \omega}(f)=x_{i_{n-m}} x_{i_{k}}$ for some $k \in\{n-m, \ldots, n-t\}$ for every $f \in \mathscr{G}$. Let $\omega^{\prime} \in \stackrel{\circ}{D}$. We now show that $\operatorname{in}_{\omega}(f)=\operatorname{in}_{\omega^{\prime}}(f)$ for every $f \in \mathscr{G}$.

If $\operatorname{in}_{\succ_{\omega}}(f)=x_{i_{j}}$ for some $j \in\{1, \ldots, n-m-1\}$, then by comparing weights, $\operatorname{in}_{\omega}(f)$ is exactly the sum of all linear terms $a_{i_{k}} x_{i_{k}}$ that appear in $f$, with $a_{i_{k}} \in K$, $k \in\{1, \ldots, n-m-1\}$. But the same is true for $\operatorname{in}_{\omega^{\prime}}(f)$, since $\omega$ and $\omega^{\prime}$ have the same minimal coordinates. So in this case $\operatorname{in}_{\omega}(f)=\operatorname{in}_{\omega^{\prime}}(f)$.

If $\operatorname{in}_{\succ_{\omega}}(f)=x_{i_{n-m}} x_{i_{k}}$ for some $k \in\{n-m, \ldots, n-t\}$, we have to distinguish two subcases.

Case 1. If $k=n-m$ or $k=n-m+1$, then $\operatorname{in}_{\omega}(f)$ is the sum of all monomials in $K\left[x_{n-m}, x_{n-m+1}\right]$ that appear in $f$. The same is true for $\operatorname{in}_{\omega^{\prime}}(f)$ by the same argument as before, so $\operatorname{in}_{\omega}(f)=\operatorname{in}_{\omega^{\prime}}(f)$.

Case 2. For $k>n-m+1$, we need to show that certain terms cannot appear in $f$. First note that no term that is divisible by any of $x_{i_{1}}, \ldots, x_{i_{n-m-1}}$ can appear in $f$, since $f$ is part of a reduced Gröbner basis with respect to $\succ_{\omega}$, and such a term would be divisible by a leading term of another element of $\mathscr{G}$. For the same reason, $f$ cannot contain the monomial $x_{i_{n-m}} x_{i_{s}}$ for $s \in\{n-m+2, \ldots, n-t\} \backslash\{k\}$. Note that $x_{i_{n-m+1}}^{2}$ cannot appear in $f$ either, since then $\mathrm{wt}_{\omega}\left(x_{i_{n-m+1}}^{2}\right)<\mathrm{wt}_{\omega}\left(x_{i_{n-m}} x_{i_{k}}\right)$. Furthermore, assume that $f$ contains the monomial $x_{i_{n-m+1}} x_{i_{s}}$ for some index $s \in$ $\{n-m+2, \ldots, n-t\} \backslash\{k\}$. Then for $v \in \mathbb{R}^{n}$ with $v_{i_{1}}=\cdots=v_{i_{n-m+1}}<v_{i_{s}}<v_{i_{j}}$ for $j \in\{n-m+2, \ldots, n\} \backslash\{k\}$, we know $\operatorname{in}_{v}(f)=x_{i_{n-m+1}} x_{i_{s}}$ is a monomial, since every other possible term of $f$ has greater $v$-weight. This is a contradiction to $v \in \mathrm{gT}(I)$. This that implies $x_{i_{n-m+1}} x_{i_{s}}$ for $s \in\{n-m+2, \ldots, n-t\} \backslash\{k\}$ does not appear in $f$ either.

With this we can determine the initial forms $\operatorname{in}_{\omega}(f)$ and $\operatorname{in}_{\omega^{\prime}}(f)$. As we have $\mathrm{wt}_{\omega}\left(x_{i_{n-m}} x_{i_{k}}\right)=\mathrm{wt}_{\omega}\left(x_{i_{n-m+1}} x_{i_{k}}\right)$, these two terms have to appear in $\operatorname{in}_{\omega}(f)$, if $x_{i_{n-m+1}} x_{i_{k}}$ is a term of $f$. Assume there exists another term in $\operatorname{in}_{\omega}(f)$; then it would have to be of the form $x_{i_{n-m}} x_{i_{r}}$ or $x_{i_{n-m+1}} x_{i_{r}}$ for some $r \in\{n-t+1, \ldots, n\}$, or of the form $x_{i_{a}} x_{i_{b}}$ for some $a, b \in\{n-m+2, \ldots, n-t\}$. The former can't occur, since $\mathrm{wt}_{\omega}\left(x_{i_{n-m}} x_{i_{r}}\right)=\mathrm{wt}_{\omega}\left(x_{i_{n-m+1}} x_{i_{r}}\right)>\mathrm{wt}_{\omega}\left(x_{i_{n-m}} x_{i_{k}}\right)$. Assume that $x_{i_{a}} x_{i_{b}}$ appears in $\operatorname{in}_{\omega}(f)$ for some $a, b \in\{n-m+2, \ldots, n-t\}$; then of course $\mathrm{wt}_{\omega}\left(x_{i_{n-m}} x_{i_{k}}\right)=$ $\mathrm{wt}_{\omega}\left(x_{i_{a}} x_{i_{b}}\right)$. But we know that $x_{i_{a}} x_{i_{b}} \succ x_{i_{n-m}} x_{i_{k}}$, by the choice of $\succ$. This is a contradiction to $\operatorname{in}_{\succ_{\omega}}(f)=x_{i_{n-m}} x_{i_{k}}$, $\operatorname{so~in}_{\omega}(f)$ only contains the monomials $x_{i_{n-m}} x_{i_{k}}$ and $x_{i_{n-m+1}} x_{i_{k}}$. 
The same is true for $\operatorname{in}_{\omega^{\prime}}(f)$, as we will see. We show that $\operatorname{in}_{\succ_{\omega^{\prime}}}(f)=x_{i_{n-m}} x_{i_{k}}$ as well. By the same argument as above, it follows that only the terms $x_{i_{n-m}} x_{i_{k}}$ and $x_{i_{n-m+1}} x_{i_{k}}$ appear in $\operatorname{in}_{\omega^{\prime}}(f)$, and thus $\operatorname{in}_{\omega}(f)=\operatorname{in}_{\omega^{\prime}}(f)$. Since $\operatorname{wt}_{\omega^{\prime}}\left(x_{i_{n-m}} x_{i_{r}}\right)=$ $\mathrm{wt}_{\omega^{\prime}}\left(x_{i_{n-m+1}} x_{i_{r}}\right)>\mathrm{wt}_{\omega^{\prime}}\left(x_{i_{n-m}} x_{i_{k}}\right)$ for $r \in\{n-t+1, \ldots, n\}$, terms of this form cannot occur as the leading term. Assume that $\operatorname{in}_{\succ_{\omega^{\prime}}}(f)=x_{i_{a}} x_{i_{b}}$ for some $a, b \in\{n-m+2$, $\ldots, n-t\}$. Then $x_{i_{a}} x_{i_{b}} \in \operatorname{gin}_{\succ_{\omega^{\prime}}}(I)$. But we know that $\operatorname{dim}\left(S / \operatorname{gin}_{\succ_{\omega^{\prime}}}(I)\right)=$ $\operatorname{dim}(S / I)=m$ and $x_{i_{1}} \succ_{\omega^{\prime}} \cdots \succ_{\omega^{\prime}} x_{i_{n-m}} \succ_{\omega^{\prime}} x_{i_{j}}$ for $j>n-m$. By Proposition 4.3, this implies that $\operatorname{gin}_{\succ_{\omega^{\prime}}}(I)$ cannot contain a monomial that is not divisible by one of $x_{i_{1}}, \ldots, x_{n-m}$, which is a contradiction to $x_{i_{a}} x_{i_{b}} \in \operatorname{gin}_{\succ_{\omega^{\prime}}}(I)$.

We have now shown that $\operatorname{in}_{\omega}(f)=\operatorname{in}_{\omega^{\prime}}(f)$ for every $f \in \mathscr{G}$. Hence, by Lemma 5.7, we have $\operatorname{in}_{\omega}(g(I))=\operatorname{in}_{\omega^{\prime}}(g(I))$ for $g \in U$. Thus every maximal cone of $\mathcal{W}_{n}^{m, t}$ is contained in a maximal cone of $\mathrm{gT}(I)$. The claim now follows from this together with Proposition 5.6.

This of course raises the question of whether it is always true that $\mathrm{gT}(I)=\mathcal{W}_{n}^{m, t}$ for strongly stable ideals or even maximal-gdepth ideals $I \subset S=K\left[x_{1}, \ldots, x_{n}\right]$ with $\operatorname{dim}(S / I)=m$ and $0<\operatorname{depth}(S / I)=t<m-1$. Computations with gfan [Jensen 2009] indicate that this is not the case. For example, the ideal $I=$ $\left(x_{1}^{2}, x_{1} x_{2}, x_{1} x_{3}^{2}, x_{1} x_{3} x_{4}\right) \subset K\left[x_{1}, \ldots, x_{5}\right]$ is strongly stable with respect to $x_{1}>$ $\cdots>x_{5}$ and has dimension $\operatorname{dim}\left(K\left[x_{1}, \ldots, x_{5}\right] / I\right)=4$ and $\operatorname{depth}\left(K\left[x_{1}, \ldots, x_{5}\right] / I\right)$ $=1$ by Proposition 4.3. However, computing $\mathrm{gT}(I)$ with gfan yields that $\mathrm{gT}(I)$ has 60 maximal cones. Thus $\operatorname{gT}(I) \neq W_{5}^{4,1}$, which has only 30 maximal cones.

Remark 5.12. If depth $(S / I)=0$, we cannot make a statement about the fan structure of the generic tropical variety of $I$. To see this, we can, for example, consider the ideals

$$
I=\left(x_{1}, \ldots, x_{n-m-1}, x_{n-m}^{2}, x_{n-m} x_{n-m+1}, \ldots, x_{n-m} x_{n}\right) \subset S=K\left[x_{1}, \ldots, x_{n}\right]
$$

for $0<m<n$, which are the ideals of Proposition 5.11 for $t=0$. By the same argument as before, we have $\operatorname{dim}(S / I)=m$ and $\operatorname{depth}(S / I)=0$. Hence, by Corollary 3.8 we know that $\mathrm{gT}(I)$ as a fan is a refinement of $\mathcal{W}_{n}^{m}$. Using the same arguments as in the proof of Proposition 5.11, we can show that $\operatorname{in}_{\omega}(g(I))=$ $\operatorname{in}_{\omega^{\prime}}(g(I))$ for $\omega, \omega^{\prime} \in \stackrel{\circ}{C}$ for any maximal cone $C$ of $\mathcal{W}_{n}^{m}$ for every $g \in U$ (as defined in (2-1)). This shows that $\mathrm{gT}(I)$ is equal to $\mathcal{W}_{n}^{m}$ as a fan.

On the other hand, we can find ideals with $\operatorname{depth}(S / I)=0$ whose generic tropical variety is a proper refinement of $\mathcal{W}_{n}^{m}$. For example, for the ideal $I=$ $\left(x_{1}^{2}, x_{1} x_{2}, x_{1} x_{3}^{2}, x_{1} x_{3} x_{4}\right) \subset K\left[x_{1}, \ldots, x_{4}\right]$ we have $\operatorname{dim}\left(K\left[x_{1}, \ldots, x_{4}\right] / I\right)=3$ and $\operatorname{depth}\left(K\left[x_{1}, \ldots, x_{4}\right] / I\right)=0$ by Proposition 4.3. If we compute $\mathrm{gT}(I)$ with gfan, however, we obtain a fan with 12 maximal cones that refines the fan $\mathcal{W}_{4}^{3}$ with only 6 maximal cones. 


\section{Multiplicities}

Let $S=K\left[x_{1}, \ldots, x_{n}\right]$ as before. For a finitely generated graded $S$-module $M$, we denote by $H_{M}(t)$ the Hilbert series of $M$. Recall that the Hilbert series of $0 \neq M$ can be written as

$$
H_{M}(t)=\frac{Q_{M}(t)}{(1-t)^{d}}
$$

where $Q_{M}(t) \in \mathbb{Z}\left[t, t^{-1}\right]$ is a Laurent polynomial with $Q_{M}(1) \neq 0$ and $d$ is the Krull dimension of $M$. It is well-known that $Q_{M}(1) \neq 0$, and this number is called the multiplicity $m(M)$ of $M$. As always, we set $m(I)=m(S / I)=Q_{S / I}(1)$ for a graded ideal $I$. We call this the multiplicity of $I$, although more precisely it is the multiplicity of $S / I$.

To express the multiplicity of $I$ in terms of the multiplicities of its minimal primes, we use the following formula, known as the associativity formula for multiplicities. Note that all minimal prime ideals of a graded ideal are graded themselves. For a minimal prime ideal $P$ of $I$, let $\ell\left((S / I)_{P}\right)$ denote the length of the localization of the $S$-module $S / I$ at $P$. We then have

$$
m(I)=\sum \ell\left((S / I)_{P}\right) m(P),
$$

with the sum taken over all minimal primes of $I$ such that $\operatorname{dim}(S / I)=\operatorname{dim}(S / P)$; see [Vasconcelos 1998, Formula (9. 4)].

We define the multiplicity of a maximal cone in $T(I)$ in a slightly more general setting than in [Dickenstein et al. 2007], where the multiplicity of a maximal cone $C$ in $T(P)$ for a prime ideal $P$ is defined as the sum of the multiplicities of all monomial-free minimal primes of the initial ideal $\operatorname{in}_{C}(P)$ corresponding to $C$. Note that by [Gräbe 1993, Theorem 1], for every minimal prime $Q$ of $\operatorname{in}_{C}(P)$, we have $\operatorname{dim}(S / Q)=\operatorname{dim}\left(S / \operatorname{in}_{C}(P)\right)$. For an arbitrary ideal $I \subset S=K\left[x_{1}, \ldots, x_{n}\right]$ this is not true, and in our definition we consider only those prime ideals $P$ of $\operatorname{in}_{C}(I)$ such that $S / P$ has the same dimension as $S /$ in $_{C}(I)$.

Definition 6.1. Let $I \subset S=K\left[x_{1}, \ldots, x_{n}\right]$ be a graded ideal and $C$ be a maximal cone of $T(I)$. Let $J=\operatorname{in}_{C}(I)$ be the initial ideal of $I$ corresponding to $C$. Then the intrinsic multiplicity $m(C)$ of $C$ is defined as $m(C)=\sum \ell\left((S / J)_{P}\right)$, where the sum is taken over all minimal primes $P$ of $J$ with $\operatorname{dim}(S / P)=\operatorname{dim}(S / J)$ that do not contain a monomial.

Note that in general $T(I)$ need not be pure, so in general this definition of intrinsic multiplicities will not give rise to a tropical fan as defined in [Gathmann et al. 2009, Definition 2.8]. However, we only need this definition for generic tropical varieties, and these are pure by Proposition 3.2. Even if $I$ is a radical ideal and $T(I)$ a pure fan, the multiplicity of the cones of $T(I)$ need not have anything to do with the multiplicity of $I$, as the following example shows. 
Example 6.2. Let $0 \leq k \leq n$ and $f_{k}=x_{1} \cdots x_{k}\left(x_{1}+x_{2}\right) \in K\left[x_{1}, \ldots, x_{n}\right]$. Then $m\left(f_{k}\right)=\operatorname{deg}\left(f_{k}\right)=k+1$. But we see that for $I=\left(f_{k}\right)$, the tropical variety

$$
T(I)=\left\{\omega \in \mathbb{R}^{n}: \omega_{1}=\omega_{2}\right\}
$$

consists of only one cone. The corresponding initial ideal is $\left(f_{k}\right)$. By factorization, this has only one monomial-free minimal prime ideal, which is $\left(x_{1}+x_{2}\right)$. Since $\ell\left(\left(S /\left(f_{k}\right)\right)_{\left(x_{1}+x_{2}\right)}\right)=1$, the only cone of $\operatorname{gT}(I)$ has multiplicity 1 . So in general it is impossible to obtain the multiplicity of the ideal from the multiplicity of the maximal cones of the tropical variety, at least for ideals that are not prime.

In contrast, we can now prove that generically, the intrinsic multiplicities of the maximal cones in the tropical variety are constant and equal to the multiplicity of the ideal. For this, we first show that for a graded ideal $I$, the minimal prime ideals of the initial ideals of $I$ that correspond to the maximal cones in $\mathrm{gT}(I)$ contain no monomial. Recall that by $U$, we denote the Zariski-open subset of $\mathrm{GL}_{n}(K)$ as defined in (2-1).

Proposition 6.3. Let $I \subset S=K\left[x_{1}, \ldots, x_{n}\right]$ be a graded ideal with $\operatorname{dim}(S / I)=m$. Let $C$ be a maximal cone of $\mathrm{gT}(I)$ and $\omega \in \stackrel{\circ}{C}$. Then no minimal prime $P$ of $\operatorname{in}_{\omega}(g(I))$ with $\operatorname{dim}(S / P)=m$ contains a monomial for $g \in U$.

Proof. Since gT $(I)=W_{n}^{m}$ as a set, we can assume $\omega_{1}=\cdots=\omega_{n-m+1}<\omega_{j}$ for $j>n-m+1$ without loss of generality. For $g \in U$, let $\operatorname{in}_{\omega}(g(I)) \subset P$ be a minimal prime ideal with $\operatorname{dim}(S / P)=m$. Assume that $P$ contains a monomial $x^{\nu}$. Since $P$ is prime, this implies that $P$ contains a variable $x_{k}$ for some $k$. We choose $\left\{i_{1}, \ldots, i_{n-m}\right\} \subset\{1, \ldots, n-m+1\} \backslash\{k\}$ and a term order $\succ$ such that

$$
x_{i_{1}} \succ x_{i_{2}} \succ \cdots \succ x_{i_{n-m}} \succ x_{j} \text { for } j \notin\left\{i_{1}, \ldots, i_{n-m}\right\} .
$$

Then

$$
\operatorname{gin}_{\succ_{\omega}}(I)=\operatorname{in}_{\succ}\left(\operatorname{in}_{\omega}(g(I))\right) \subset \operatorname{in}_{\succ}(P),
$$

with $\operatorname{dim}\left(S / \operatorname{gin}_{\succ_{\omega}}(I)\right)=\operatorname{dim}\left(S / \operatorname{in}_{\succ}(P)\right)=m$. Let $Q$ be a minimal prime of $\operatorname{in}_{\succ}(P)$. Since the dimensions coincide, $Q$ is also a minimal prime of $\operatorname{gin}_{\succ_{\omega}}(I)$. But $\operatorname{gin}_{\succ_{\omega}}(I)$ has only one minimal prime, which is $\left(x_{i_{1}}, \ldots, x_{i_{n-m}}\right)$ by the choice of the term order $\succ$; see for example [Eisenbud 1995, Corollary 15.25]. Hence, $Q$ does not contain $x_{k}$. This is a contradiction to the fact that $x_{k} \in P$, and therefore $x_{k} \in \operatorname{in}_{\succ}(P) \subset Q$. Thus, $P$ cannot contain a monomial.

Remark 6.4. Note that together with [Römer and Schmitz 2009, Lemma 7.2], where $\mathrm{gT}(I)$ can be replaced by $T(g(I))$ for every $g \in U$, and [Römer and Schmitz 2009, Corollary 3.2], this gives another, simpler proof that generic tropical varieties exist as described in [Römer and Schmitz 2009]. 
To use the associativity formula for multiplicities to show that $m(C)=m(I)$ in generic tropical varieties, we need to show that generically all minimal primes of $\operatorname{in}_{\omega}(g(I))$ have multiplicity 1 . This we do by showing that they are linear, that is, generated by linear forms.

Lemma 6.5. Let $P \subset K\left[x_{1}, \ldots, x_{n}\right]$ be a graded prime ideal with $\operatorname{dim}(S / P)=1$. Then $P$ is a linear ideal.

Proof. As $P \neq\left(x_{1}, \ldots, x_{n}\right)$, we know that $V(P) \neq\{0\}$. Let

$$
0 \neq a=\left(a_{1}, \ldots, a_{n}\right) \in V(P) \subset K^{n} .
$$

Then $V(Q)=K\left(a_{1}, \ldots, a_{n}\right)$ for the linear ideal $Q=\left(a_{i} x_{j}-a_{j} x_{i}: i<j\right)$. Since $V(Q) \subset V(P)$ and both are prime, this implies $P \subset Q \subset\left(x_{1}, \ldots, x_{n}\right)$. But $\operatorname{dim}(S / P)=1$ and $Q \neq\left(x_{1}, \ldots, x_{n}\right)$, hence $P=Q$ is linear.

Lemma 6.6. For a fixed $t<n$, let $R=K\left[x_{1}, \ldots, x_{t}\right]$. Let $J \subset S=K\left[x_{1}, \ldots, x_{n}\right]$ be a graded ideal and $J \subset P \subset S$ be a minimal prime of $J$ with $\operatorname{dim}(S / J)=$ $\operatorname{dim}(S / P)=m$. If $(J \cap R) S=J$, then also $(P \cap R) S=P$.

Proof. It is clear that $(P \cap R) S \subset P$. As $J \subset P$, we know that $J=(J \cap R) S \subset$ $(P \cap R) S \subset P$. Since $P$ is prime, so are $P \cap R$ and $(P \cap R) S$. But $P$ is a minimal prime of $J$, and hence $(P \cap R) S=P$.

With this we can prove that for $I \subset K\left[x_{1}, \ldots, x_{n}\right]$, the minimal primes of the initial ideals corresponding to the maximal cones of $\mathrm{gT}(I)$ of the same dimension as $S / I$ have multiplicity 1 .

Proposition 6.7. Let $I \subset S=K\left[x_{1}, \ldots, x_{n}\right]$ be a graded ideal with $\operatorname{dim}(S / I)=m$ and $\omega \in \stackrel{\circ}{C}$ for some maximal cone $C$ of $\mathrm{gT}(I)$. Then for every $g \in U$, every minimal prime $P$ of $\operatorname{in}_{\omega}(g(I))$ with $\operatorname{dim}(S / P)=\operatorname{dim}\left(S / \operatorname{in}_{\omega}(g(I))\right)$ is a linear ideal. In particular, $m(P)=1$.

Proof. Without loss of generality we can assume $\omega_{1}=\cdots=\omega_{n-m+1}<\omega_{j}$ for $j>n-m+1$. Let $g \in U$, and let $\operatorname{in}_{\omega}(g(I)) \subset P$ be a minimal prime with $\operatorname{dim}(S / P)=m$. Let $\mathscr{G}=\left\{f_{1}, \ldots, f_{t}\right\}$ be a reduced Gröbner basis of $g(I)$ with respect to $\succ_{\omega}$ for a term order $\succ$ with $x_{1} \succ \cdots \succ x_{n}$. Then

$$
\left.\operatorname{in}_{\succ \omega}\left(f_{i}\right): i=1, \ldots, t\right)=\operatorname{in}_{\succ}\left(\operatorname{in}_{\omega}(g(I))\right)=\operatorname{gin}_{\succ_{\omega}}(I) .
$$

Note that $x_{1} \succ_{\omega} \cdots \succ_{\omega} x_{n-m+1} \succ_{\omega} x_{j}$ for $j>n-m+1$. Let $A \subset\{1, \ldots, t\}$ be the set of all indices $i$ such that $\operatorname{in}_{\succ_{\omega}}\left(f_{i}\right) \in K\left[x_{1}, \ldots, x_{n-m}\right]$. We define $\tilde{J}=\left(\operatorname{in}_{\succ_{\omega}}\left(f_{i}\right)\right.$ : $i \in A$ ) to be the ideal generated by all initial forms of elements in $G_{G}$ that are not divisible by $x_{n-m+1}, \ldots, x_{n}$. Since $\tilde{J} \subset \operatorname{gin}_{\succ_{\omega}}(I)$, we know that $\operatorname{dim}(S / \tilde{J}) \geq m$. As $\operatorname{gin}_{\succ_{\omega}}(I)$ is a strongly stable ideal, by Proposition 4.3 there exists $1 \leq k \leq t$ such that $\operatorname{in}_{\succ_{\omega}}\left(f_{k}\right)=x_{n-m}^{d}$ for some $d \in \mathbb{N}$. Hence, $x_{n-m}^{d} \in \tilde{J}$. But $\tilde{J}$ is also a 
strongly stable ideal, so again by Proposition 4.3 it follows that $\operatorname{dim}(S / \tilde{J}) \leq m$. Thus, $\operatorname{dim}(S / \tilde{J})=m$. We set $J=\left(\operatorname{in}_{\omega}\left(f_{i}\right): i \in A\right)$. Then we have

$$
m=\operatorname{dim}\left(S / \operatorname{in}_{\omega}(g(I))\right) \leq \operatorname{dim}(S / J)=\operatorname{dim}\left(S / \operatorname{in}_{\succ}(J)\right) \leq \operatorname{dim}(S / \tilde{J})=m,
$$

where the first inequality holds because $J \subset \operatorname{in}_{\omega}(g(I))$, and the second because $\tilde{J} \subset \operatorname{in}_{\succ}(J)$. So $\operatorname{dim}(S / J)=m$. Since $J \subset \operatorname{in}_{\omega}(g(I)) \subset P$ and all considered rings have the same dimension, $P$ is also a minimal prime ideal of $J$.

Let $R$ be the polynomial ring $K\left[x_{1}, \ldots, x_{n-m+1}\right]$, so $S=R\left[x_{n-m+2}, \ldots, x_{n}\right]$. For $i \in A$, every term of $f_{i}$ that has minimal $\omega$-weight has to be a term in $R$ by the choice of $\omega$. So we know that $J=(J \cap R) S$. From Lemma 6.6 it now follows that $P=(P \cap R) S$. Let $\tilde{P}=P \cap R$. Then we have

$$
S / P=S /(\tilde{P} S) \cong R / \tilde{P}\left[x_{n-m+2}, \ldots, x_{n}\right] .
$$

Hence, $\operatorname{dim}(S / P)=\operatorname{dim}(R / \tilde{P})+(m-1)$, so $R / \tilde{P}$ has dimension $m-(m-1)=1$ in $R$. By Lemma 6.5, we know that $\tilde{P}$ is linear. So $P=\tilde{P} S$ is linear as well and in particular, $m(P)=1$.

Theorem 6.8. Let $I \subset S=K\left[x_{1}, \ldots, x_{n}\right]$ be a graded ideal with $\operatorname{dim}(S / I)=m$. Then for $g \in U$ and any maximal cone $C$ of $T(g(I))$, we have $m(C)=m(I)$, so the intrinsic multiplicity of every maximal cone equals the multiplicity of $I$.

Proof. First note that the Hilbert series and thus the multiplicity of $I$ does not change if one passes to any initial ideal of $I$; see for example [Eisenbud 1995, Theorem 15.26]. Moreover, the Hilbert series is of course not affected by a coordinate change.

By Proposition 6.3, for $g \in U$ and any maximal cone $C$ of $T(g(I))=\mathrm{gT}(I)$, we know that every minimal prime $P$ of $\operatorname{in}_{C}(g(I))$ with $\operatorname{dim}(S / P)=m$ does not contain a monomial. Moreover, by Proposition 6.7, every such minimal prime $P$ of $\operatorname{in}_{C}(g(I))$ has multiplicity $m(P)=1$. Thus with the associativity formula for multiplicities, we get

$$
\begin{aligned}
m(C)=\sum \ell\left(\left(S / \operatorname{in}_{C}(g(I))\right)_{P}\right) & =\sum \ell\left(\left(S / \operatorname{in}_{C}(g(I))\right)_{P}\right) m(P) \\
& =m\left(\operatorname{in}_{C}(g(I))\right)=m(I),
\end{aligned}
$$

as the sum is taken over all minimal primes of $\operatorname{in}_{C}(g(I))$ with $\operatorname{dim}(S / P)=m$.

Remark 6.9. The fan $\mathrm{gT}(I)$ equipped with the weights $m(C)$ for the maximal cones $C \in \mathrm{gT}(I)$ is a tropical fan in the sense of [Gathmann et al. 2009, Definition 2.8]. It can be shown directly by elementary methods that the balancing condition is fulfilled for each cone of dimension $\operatorname{dim}(S / I)-1$. See [Speyer 2005, Theorem 2.5.1] for a proof in a more general case. 
We briefly explain [Dickenstein et al. 2007, Example (1)] in our case. This example states that for an irreducible polynomial $f \in K\left[x_{1}, \ldots, x_{n}\right]$, the intrinsic multiplicity $m(C)$ of a given cone $C$ of $T(f)$ is exactly the lattice length of the edge corresponding to $C$ in the Newton polytope of $f$. Here, the lattice length of an edge is defined as the number of integer points on this edge minus 1 .

Example 6.10. Let $0 \neq f \in K\left[x_{1}, \ldots, x_{n}\right]$ be a homogeneous polynomial of degree $t$. Then every maximal cone of $\operatorname{gT}(f)$ has multiplicity $t$, as $m(g(f))=\operatorname{deg} g(f)=t$ for every $g \in U$. Let $N(g(f))$ be the Newton polytope of $g(f)$ for $g \in U$. By [Römer and Schmitz 2009, Lemma 8.5], for $g \in U$ we know that

$$
N(g(f))=\operatorname{conv}\left(t e_{1}, \ldots, t e_{n}\right),
$$

where $e_{1}, \ldots, e_{n}$ are the standard basis vectors in $\mathbb{R}^{n}$. Now a maximal cone $C$ of $\mathrm{gT}(f)$ is given by

$$
C=\left\{\omega \in \mathbb{R}^{n}: \omega_{i_{1}}=\omega_{i_{2}} \leq \omega_{i_{j}} \text { for } j \neq 1,2\right\}
$$

for some coordinates $i_{1}, i_{2}$. This corresponds to the edge $\operatorname{conv}\left(t e_{i_{1}}, t e_{i_{2}}\right)$ of $N(g(f))$ for $g \in U$. This edge has lattice length $t$, that is, $\left|\left\{\mathbb{Z}^{n} \cap \operatorname{conv}\left(t e_{i_{1}}, t e_{i_{2}}\right)\right\}\right|=t+1$. So the lattice length coincides with the intrinsic multiplicity $m(C)$.

\section{Acknowledgments}

We thank Hannah Markwig and Bernd Sturmfels for useful suggestions for this paper, and we are especially grateful to Diane Maclagan for many illuminating discussions.

\section{References}

[Bieri and Groves 1984] R. Bieri and J. R. J. Groves, "The geometry of the set of characters induced by valuations", J. Reine Angew. Math. 347 (1984), 168-195. MR 86c:14001 Zbl 0526.13003

[Bogart et al. 2007] T. Bogart, A. N. Jensen, D. Speyer, B. Sturmfels, and R. R. Thomas, "Computing tropical varieties”, J. Symbolic Comput. 42:1-2 (2007), 54-73. MR 2007j:14103 Zbl 1121.14051

[Bruns and Conca 2004] W. Bruns and A. Conca, "Gröbner bases, initial ideals and initial algebras", in Homological methods in commutative algebra (Tehran, 2004), edited by L. L. Avramov et al., 2004.

[Bruns and Gubeladze 2009] W. Bruns and J. Gubeladze, Polytopes, rings, and K-theory, Springer, Dordrecht, 2009. MR 2010d:19001 Zbl 1168.13001

[Bruns and Herzog 1993] W. Bruns and J. Herzog, Cohen-Macaulay rings, Cambridge Studies in Advanced Mathematics 39, Cambridge University Press, 1993. MR 95h:13020 Zbl 0788.13005

[Develin and Sturmfels 2004] M. Develin and B. Sturmfels, "Tropical convexity", Doc. Math. 9 (2004), 1-27. Erratum in 9 (2004), 205-206. MR 2005i:52010 Zbl 1054.52004

[Dickenstein et al. 2007] A. Dickenstein, E. M. Feichtner, and B. Sturmfels, "Tropical discriminants”, J. Amer. Math. Soc. 20:4 (2007), 1111-1133. MR 2008j:14095 Zbl 1166.14033 
[Draisma 2008] J. Draisma, "A tropical approach to secant dimensions", J. Pure Appl. Algebra 212:2 (2008), 349-363. MR 2008j:14102 Zbl 1126.14059

[Eisenbud 1995] D. Eisenbud, Commutative algebra, Graduate Texts in Mathematics 150, Springer, New York, 1995. MR 97a:13001 Zbl 0819.13001

[Eisenbud and Goto 1984] D. Eisenbud and S. Goto, "Linear free resolutions and minimal multiplicity”, J. Algebra 88:1 (1984), 89-133. MR 85f:13023 Zbl 0531.13015

[Eliahou and Kervaire 1990] S. Eliahou and M. Kervaire, "Minimal resolutions of some monomial ideals", J. Algebra 129:1 (1990), 1-25. MR 91b:13019 Zbl 0701.13006

[Gathmann 2006] A. Gathmann, “Tropical algebraic geometry”, Jahresber. Deutsch. Math.-Verein. 108:1 (2006), 3-32. MR 2007e:14088 Zbl 1109.14038

[Gathmann and Markwig 2008] A. Gathmann and H. Markwig, "Kontsevich's formula and the WDVV equations in tropical geometry”, Adv. Math. 217:2 (2008), 537-560. MR 2370275 Zbl 1131.14057

[Gathmann et al. 2009] A. Gathmann, M. Kerber, and H. Markwig, "Tropical fans and the moduli spaces of tropical curves", Compos. Math. 145:1 (2009), 173-195. MR 2009m:14085 Zbl 1169. 51021

[Gräbe 1993] H.-G. Gräbe, “Two remarks on independent sets”, J. Algebraic Combin. 2:2 (1993), 137-145. MR 94e:13051

[Herzog and Srinivasan 1998] J. Herzog and H. Srinivasan, "Bounds for multiplicities", Trans. Amer. Math. Soc. 350:7 (1998), 2879-2902. MR 99g:13033 Zbl 0899.13026

[Itenberg et al. 2007] I. Itenberg, G. Mikhalkin, and E. Shustin, Tropical algebraic geometry, Oberwolfach Seminars 35, Birkhäuser, Basel, 2007. MR 2008e:14082 Zbl 1162.14300

[Jensen 2007] A. N. Jensen, Algorithmic aspects of Gröbner fans and tropical varieties, Ph.D. thesis, Aarhus University, 2007, Available at http://tinyurl.com/2ehs2ho.

[Jensen 2009] A. N. Jensen, "Gfan, a software system for Gröbner fans and tropical varieties", version 0.4, 2009, Available at http://www.math.tu-berlin.de/ jensen/software/gfan/gfan.html.

[Jensen et al. 2008] A. N. Jensen, H. Markwig, and T. Markwig, "An algorithm for lifting points in a tropical variety”, Collect. Math. 59:2 (2008), 129-165. MR 2009a:14077 Zbl 1151.13021

[Katz et al. 2008] E. Katz, H. Markwig, and T. Markwig, "The $j$-invariant of a plane tropical cubic", J. Algebra 320:10 (2008), 3832-3848. MR 2010b:14122 Zbl 1185.14030

[Maclagan and Thomas 2007] D. Maclagan and R. R. Thomas, "Computational algebra and combinatorics of toric ideals", pp. 1-106 in Commutative algebra and combinatorics (Allahabad, 2003), vol. 1, edited by R. V. Gurjar et al., Ramanujan Math. Soc. Lect. Notes Ser. 4, Ramanujan Math. Soc., Mysore, 2007. MR 2009c:13069

[Mikhalkin 2006] G. Mikhalkin, “Tropical geometry and its applications”, pp. 827-852 in International Congress of Mathematicians (Madrid, 2006), vol. 2, edited by M. Sanz-Solé et al., Eur. Math. Soc., Zürich, 2006. MR 2008c:14077 Zbl 1103.14034

[Mora and Robbiano 1988] T. Mora and L. Robbiano, "The Gröbner fan of an ideal”, J. Symbolic Comput. 6:2-3 (1988), 183-208. MR 90d:13004 Zbl 0668.13017

[Römer and Schmitz 2009] T. Römer and K. Schmitz, "Generic tropical varieties”, preprint, 2009. arXiv 0904.0120

[Speyer 2005] D. E. Speyer, Tropical geometry, ProQuest LLC, Ann Arbor, MI, 2005. MR 2623018

[Speyer and Sturmfels 2004] D. Speyer and B. Sturmfels, "The tropical Grassmannian", Adv. Geom. 4:3 (2004), 389-411. MR 2005d:14089 Zbl 1065.14071 
[Sturmfels 1996] B. Sturmfels, Gröbner bases and convex polytopes, University Lecture Series 8, American Mathematical Society, Providence, RI, 1996. MR 97b:13034 Zbl 0856.13020

[Vasconcelos 1998] W. V. Vasconcelos, Computational methods in commutative algebra and algebraic geometry, Algor. Comput. Math. 2, Springer, Berlin, 1998. MR 99c:13048 Zbl 0896.13021

Communicated by Joseph Gubeladze

Received 2009-09-11 Revised 2010-02-05 Accepted 2010-04-06

troemer@uos.de

Institut für Mathematik, Universität Osnabrück, 49069 Osnabrück, Germany

kischmit@uos.de

Institut für Mathematik, Universität Osnabrück, 49069 Osnabrück, Germany 


\section{Algebra \& Number Theory}

www.jant.org

\section{EDITORS}

\section{MANAGING EDITOR}

Bjorn Poonen

Massachusetts Institute of Technology

Cambridge, USA

\author{
EDITORIAL BOARD CHAIR \\ David Eisenbud \\ University of California \\ Berkeley, USA
}

\section{BOARD OF EDITORS}

\section{Georgia Benkart}

Dave Benson

Richard E. Borcherds

John H. Coates

J-L. Colliot-Thélène

Brian D. Conrad

Hélène Esnault

Hubert Flenner

Edward Frenkel

Andrew Granville

Joseph Gubeladze

Ehud Hrushovski

Craig Huneke

Mikhail Kapranov

Yujiro Kawamata

János Kollár

Hendrik W. Lenstra

Yuri Manin

Barry Mazur
University of Wisconsin, Madison, USA

University of Aberdeen, Scotland

University of California, Berkeley, USA

University of Cambridge, UK

CNRS, Université Paris-Sud, France

University of Michigan, USA

Universität Duisburg-Essen, Germany

Ruhr-Universität, Germany

University of California, Berkeley, USA

Université de Montréal, Canada

San Francisco State University, USA

Hebrew University, Israel

University of Kansas, USA

Yale University, USA

University of Tokyo, Japan

Princeton University, USA

Universiteit Leiden, The Netherlands

Northwestern University, USA

Harvard University, USA
Susan Montgomery

Shigefumi Mori

Andrei Okounkov

Raman Parimala

Victor Reiner

Karl Rubin

Peter Sarnak

Michael Singer

Ronald Solomon

Vasudevan Srinivas

J. Toby Stafford

Bernd Sturmfels

Richard Taylor

Ravi Vakil

Michel van den Bergh

Marie-France Vignéras

Kei-Ichi Watanabe

Andrei Zelevinsky

Efim Zelmanov
University of Southern California, USA

RIMS, Kyoto University, Japan

Princeton University, USA

Emory University, USA

University of Minnesota, USA

University of California, Irvine, USA

Princeton University, USA

North Carolina State University, USA

Ohio State University, USA

Tata Inst. of Fund. Research, India

University of Michigan, USA

University of California, Berkeley, USA

Harvard University, USA

Stanford University, USA

Hasselt University, Belgium

Université Paris VII, France

Nihon University, Japan

Northeastern University, USA

University of California, San Diego, USA

\section{PRODUCTION}

ant@mathscipub.org

Paulo Ney de Souza, Production Manager

Silvio Levy, Senior Production Editor

See inside back cover or www.jant.org for submission instructions.

The subscription price for 2010 is US \$140/year for the electronic version, and \$200/year (+\$30 shipping outside the US) for print and electronic. Subscriptions, requests for back issues from the last three years and changes of subscribers address should be sent to Mathematical Sciences Publishers, Department of Mathematics, University of California, Berkeley, CA 94720-3840, USA.

Algebra \& Number Theory (ISSN 1937-0652) at Mathematical Sciences Publishers, Department of Mathematics, University of California, Berkeley, CA 94720-3840 is published continuously online. Periodical rate postage paid at Berkeley, CA 94704, and additional mailing offices.

ANT peer-review and production is managed by EditFLOw ${ }^{\mathrm{TM}}$ from Mathematical Sciences Publishers.

PUBLISHED BY

mathematical sciences publishers

http://www.mathscipub.org

A NON-PROFIT CORPORATION

Typeset in LATEX

Copyright $\odot 2010$ by Mathematical Sciences Publishers 


\section{Algebra \& Number Theory}

Volume $4 \quad$ No. $4 \quad 2010$

Stable reduction of $X_{0}\left(p^{3}\right)$

Ken MCMurdy and Robert Coleman

Cyclotomic function fields, Artin-Frobenius automorphisms, and list error correction with optimal rate

VENKATESAN GURUSWAMI

Algebraic properties of generic tropical varieties

TIM RÖMER and KIRSTEN SCHMITZ 